\title{
Exosomes from mesenchymal stromal cells reduce murine colonic inflammation via a macrophage-dependent mechanism
}

\author{
Huashan Liu, ${ }^{1,2}$ Zhenxing Liang, ${ }^{1,2}$ Fengwei Wang, ${ }^{3}$ Chi Zhou, ${ }^{1}$ Xiaobin Zheng, ${ }^{1}$ Tuo Hu, ${ }^{1}$ Xiaowen He, \\ Xianrui $\mathrm{Wu}^{1,2}$ and Ping Lan ${ }^{1,2}$ \\ 'Department of Colorectal Surgery and Guangdong Provincial Key Laboratory of Colorectal and Pelvic Floor Diseases, The \\ Sixth Affiliated Hospital, Sun Yat-sen University, Guangzhou, Guangdong, China. ${ }^{2}$ Guangzhou Regenerative Medicine \\ and Health Guangdong Laboratory, Guangzhou, China. ${ }^{3}$ State Key Laboratory of Oncology in South China, Collaborative \\ Innovation Center for Cancer Medicine, Sun Yat-sen University Cancer Center, Guangzhou, Guangdong, China.
}

Conventional treatments for inflammatory bowel disease (IBD) have multiple potential side effects. Therefore, alternative treatments are desperately needed. This work demonstrated that systemic administration of exosomes from human bone marrow-derived mesenchymal stromal cells (MSC-Exos) substantially mitigated colitis in various models of IBD. MSC-Exos treatment downregulated inflammatory responses, maintained intestinal barrier integrity, and polarized M2b macrophages but did not favor intestinal fibrosis. Mechanistically, infused MSC-Exos acted mainly on colonic macrophages, and macrophages from colitic colons acquired obvious resistance to inflammatory restimulation when prepared from mice treated with MSC-Exos versus untreated mice. The beneficial effect of MSC-Exos was blocked by macrophage depletion. Also, the induction of IL-10 production from macrophages was partially involved in the beneficial effect of MSC-Exos. MSC-Exos were enriched in proteins involved in regulating multiple biological processes associated with the anticolitic benefit of MSC-Exos. Particularly, metallothionein-2 in MSC-Exos was required for the suppression of inflammatory responses. Taken together, MSC-Exos are critical regulators of inflammatory responses and may be promising candidates for IBD treatment.

Authorship note: $\mathrm{HL}, \mathrm{ZL}$, and FW contributed equally to this work.

Conflict of interest: The authors have declared that no conflict of interest exists.

Copyright: (c) 2019, American Society for Clinical Investigation.

Submitted: June 24, 2019

Accepted: October 31, 2019

Published: December 19, 2019

Reference information: JCl Insight. 2019;4(24):e131273.

https://doi.org/10.1172/jci.

insight.131273.

\section{Introduction}

Inflammatory bowel disease (IBD) is a family of chronic, idiopathic, relapsing, and tissue-destructive disorders, including Crohn's disease and ulcerative colitis, that has emerged as a worldwide public health challenge (1). It is characterized by an abnormal immune response to the gut microflora in genetically susceptible individuals (2). Despite advances in antitumor necrosis factor therapy, management of IBD in current clinical practice remains a daunting challenge (3). Traditional therapies for IBD frequently result in multiple serious side effects and treatment resistance (4), highlighting the unmet need for alternative treatment options for IBD.

Mesenchymal stromal cells (MSCs) have recently shown great potential as a feasible and effective biological treatment for IBD (5-7). The antiinflammatory and immunomodulatory activities of MSCs provide the theoretical grounds for MSC transplantation in IBD patients and as a potential novel cellular therapy for IBD (8). Several preclinical studies demonstrate that implanted MSCs can home to the injured tissue and differentiate as functional cells in situ to replace damaged cells (9). However, only a low percentage of implanted MSCs survive in vivo and engraft into injured tissues (10). This strongly suggests that therapeutic effects mediated by MSCs might be attributed to their secretory pathways rather than their tissue-homing capacity. Indeed, the cell-free conditioned media of MSCs and the secretome of MSCs (containing extracellular vesicles and exosomes) afford beneficial effects in various diseases (11-13). Despite numerous therapeutic vectors in the MSC secretome with antiinflammatory and immunosuppressive properties, no specific mediators have been identified as responsible for the beneficial effects of MSC-based therapy. Moreover, the risk of malignant transformation in human recipients, though not yet definitely confirmed, remains a critical problem impeding progress in the clinical translation of MSCs $(5,14)$. 
In contrast, exosomes derived from MSCs (MSC-Exos), providing immune regulatory and tissue repair properties like MSCs, have recently emerged as a powerful component in the "secretory pathways" of MSCs and have aroused great enthusiasm (15). Indeed, MSC-Exos evoke MSC-like protective effects in several diseases (16-18). Mechanistically, Zhao et al. recently reported that exosomes from adipose-derived stem cells attenuate adipose inflammation and obesity through polarizing M2 macrophages (19). In addition, Willis et al. demonstrated that MSC-Exos blunt hyperoxia-induced inflammation, partially by modulating the lung macrophage phenotype (11). MSC-Exos-based cell-free treatment is more than a compensation for MSC-based treatment. MSC-Exos possess certain desirable features, including immunosilence, non-oncogenicity, high stability, cell- and tissue-specific homing, and no vascular obstructive propensity (20, 21). Therefore, MSC-Exos offer a therapeutic advantage over MSC-based treatment, and it is of increasing interest to explore the potential roles of MSC-Exos in the management of IBD and their related mechanisms. Accordingly, we show that human bone marrow-derived MSC-Exos have protective effects on experimental colitis. Furthermore, we characterize the biological mechanism underlying the therapeutic value of MSC-Exos. Our findings present a potentially new therapeutic tool for the treatment of IBD.

\section{Results}

Isolation and purification of MSC-Exos. We isolated MSC-Exos from the supernatants of human bone marrow-derived MSCs. Before exosome extraction, flow cytometric analysis for MSC-related markers revealed that the MSCs were positive ( $\geq 95 \%$ ) for CD105, CD90, and CD73 but were negative $(\leq 2 \%)$ for CD45, CD79a, CD19, CD34, CD14, CD11b, and HLA-DR (Figure 1A). Propidium iodide (PI) and Annexin V analysis showed a good in vitro culture condition of the MSCs (Figure 1B), ruling out apoptotic bodies and random cell debris. Particle metrix (PMX) (Figure 1C) and transmission electron microscopy (TEM) (Figure 1D) confirmed their size distribution and shape were consistent with exosomes. Furthermore, Western blot revealed the exosomal identity markers TSG101 and CD9 (Figure 1E). Together, these data are indicative of successful isolation and purification of MSC-Exos.

MSC-Exos inhibit chemically induced colitis in mice. Next, we explored the potential protective effects of MSC-Exos in murine models of IBD induced by oral dextran sodium sulfate (DSS) administration or intrarectal infusion of 2,4,6-trinitrobenzenesulfonic acid solution (TNBS), both of which exhibit clinical, histopathological, and immunological phenotypes that closely parallel human IBD. By 7 days, mice receiving oral administration of 5\% DSS displayed significantly elevated disease activity indexes (DAIs) (Figure 2A), with continuous body weight loss (Figure 2B). In striking contrast, intravenous injection of MSC-Exos reduced the clinical activity compared with that in mice treated with DSS plus phosphate-buffered saline (PBS) (Figure 2, A and B). Simultaneously, DSS administration led to significant colon shortenings, whereas MSC-Exos treatment significantly improved colonic inflammation, as shown by an increased colon length (Figure 2C). Histological examination indicated that MSC-Exos treatment maintained intestinal structural integrity and significantly reduced disruptions of the architecture, crypt loss, and infiltration of inflammatory cells, with lower histological scores compared with corresponding controls (Figure 2, D and E). Moreover, MSC-Exos treatment afforded a comparable decrease in colonic myeloperoxidase (MPO) activity, suggesting less neutrophil infiltration (Figure $2 \mathrm{~F}$ ).

We further examined the anticolitic benefit of MSC-Exos in chronic and recurrent colitis. Oral treatment of $3 \%$ DSS in 2 cycles resulted in 2 peaks of colitis characterized by continuous weight loss, diarrhea, and bloody stools and causing $\sim 80 \%$ mortality (Figure 3, A and B). MSC-Exos infusion at the first acute peak of colitis (day 7) markedly ameliorated the disease severity by day 9 , as demonstrated by improved survival and stool consistency, less rectal bleeding, decreased colon shortening, reduced MPO activity, and amelioration of colon damage (Figure 3, A-F). Noteworthily, mice with a single MSC-Exos injection at the first disease peak were endowed with clear resistance to disease activity during the second cycle of DSS treatment. A second MSC-Exos treatment at the second onset of disease (day 16) effectively prevented the recurrence of colitis (Figure 3, A-F).

The anticolitic benefit of MSC-Exos was not limited to DSS-induced colitis. Analogous to results using the DSS-induced colitis model, MSC-Exos were shown to be effective in inhibiting TNBSinduced colitis (Supplemental Figure 1, A-F). Moreover, the therapeutic effects of MSC-Exos on colitis seemed to be dose and frequency dependent (Supplemental Figure 2, A and B). However, pretreatment with MSC-Exos 2 days before DSS administration did not provide prophylactic effects for DSS-induced colitis (Supplemental Figure 2, C and D). 
A
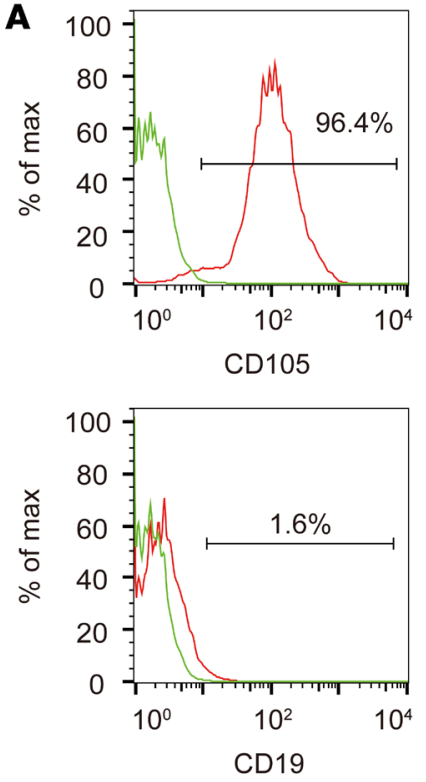

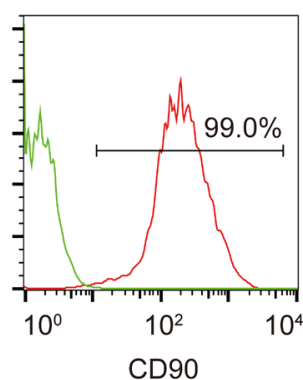

CD90

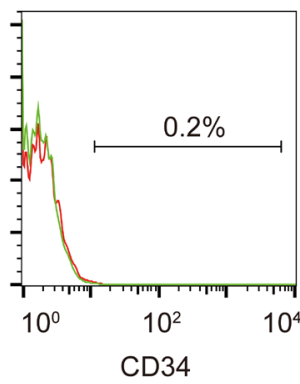

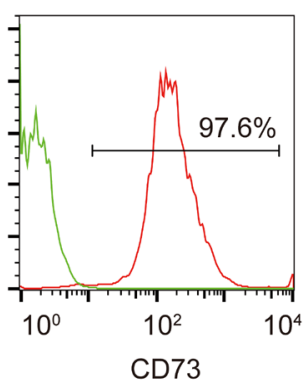

CD73

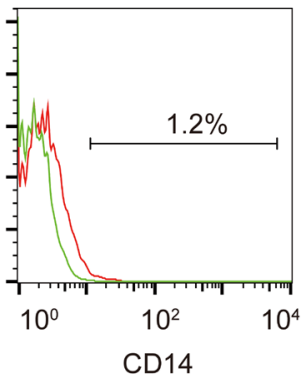

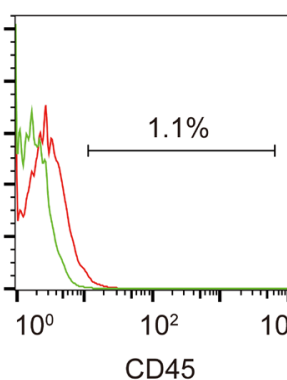
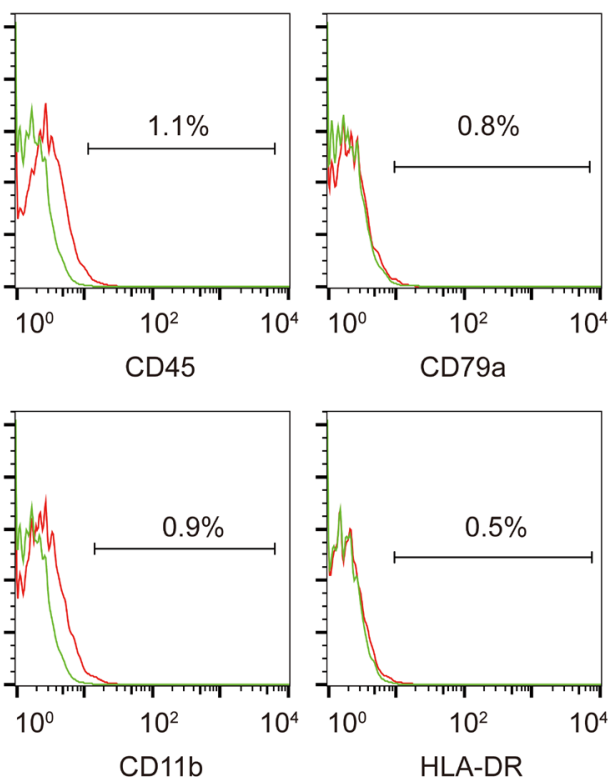

B

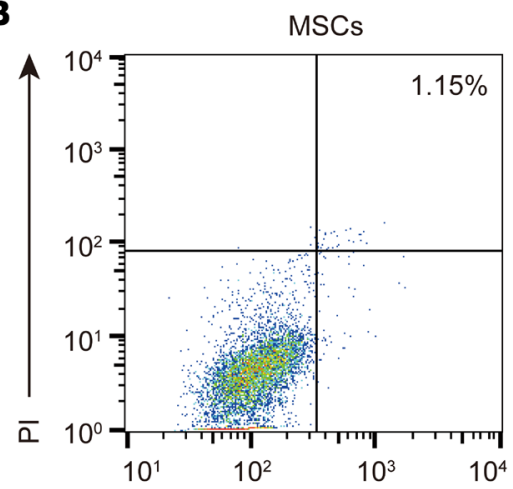

Annexin V
MSCs treated with etoposide

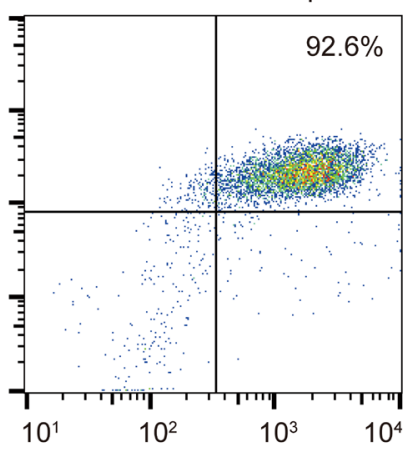

D

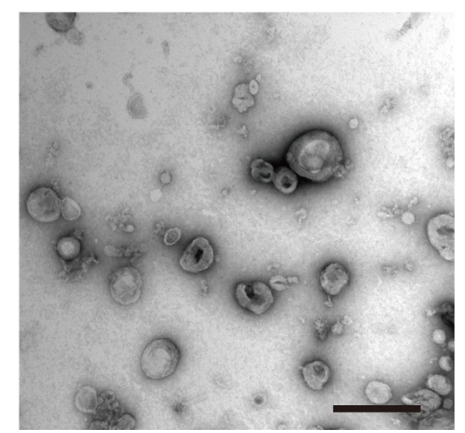

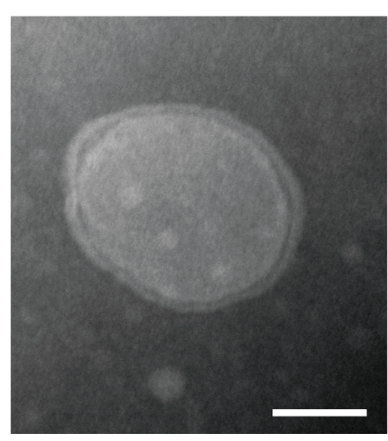

C

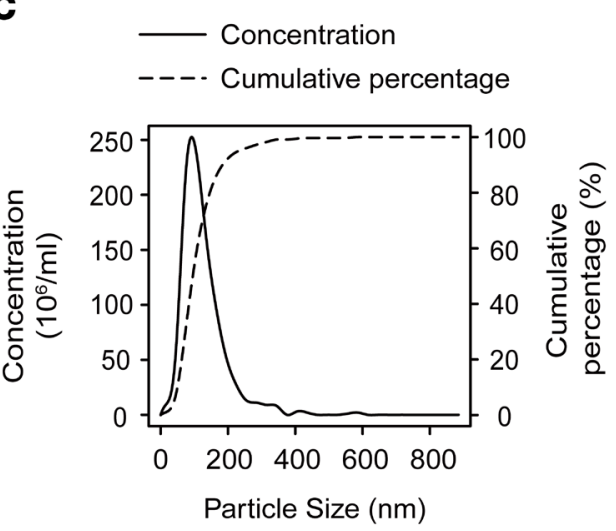

E

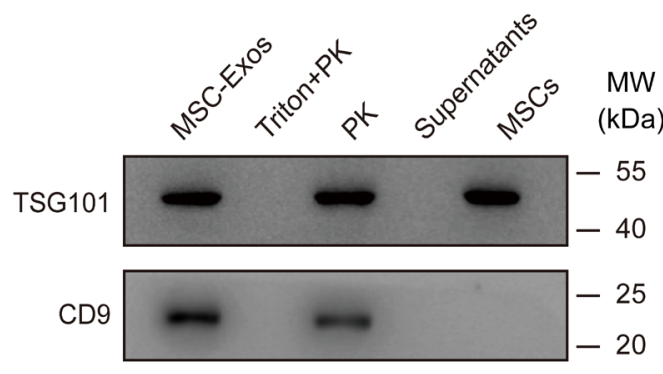

Figure 1. Characterization of MSC-Exos. Three independent experiments were performed and yielded similar results. (A) Flow cytometry analysis showing the phenotypic markers of passage 5 MSCs cultured in medium supplemented with $10 \%$ exosome-depleted fetal bovine serum (FBS). Numerical values denote the percentage of positive cells. (B) Flow cytometry analysis for PI and Annexin V of MSCs cultured in exosome-depleted FBS medium. The apoptosis of MSCs was induced by $10 \mu \mathrm{M}$ etoposide as a positive control. Numerical values denote the percentage of apoptotic cells $\left(\right.$ Annexin $\mathrm{V}^{+}$). (C) Size profile of MSC-Exos by PMX. (D) TEM analysis of MSC-Exos. TEM using negative staining with uranyl acetate (left) and tungstophosphoric acid (right). Scale bars: $200 \mathrm{~nm}$ (left), $50 \mathrm{~nm}$ (right). (E) Western blot analysis of TSC101 and CD9. Extracts of MSC-Exos were exposed to Triton X-100 plus proteinase K (PK) or PK alone. MW, molecular weight. 
A
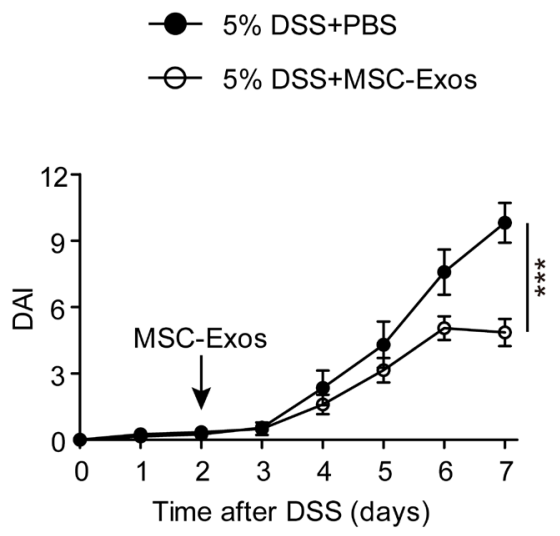

D

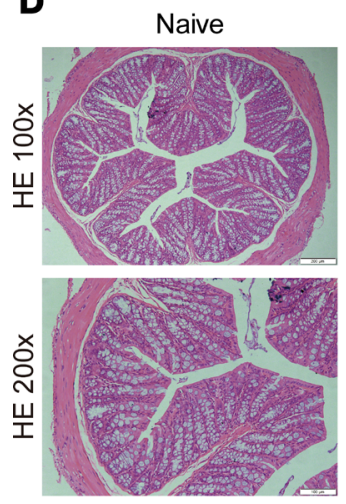

B
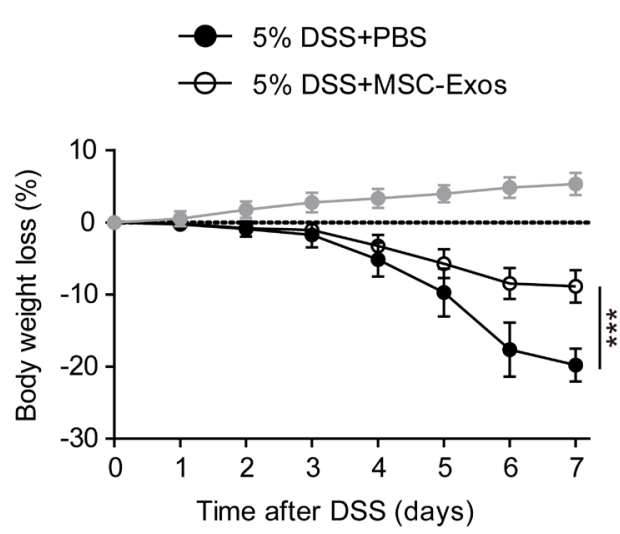

E

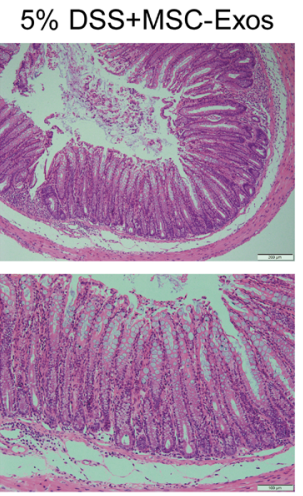

$5 \%$ DSS+PBS

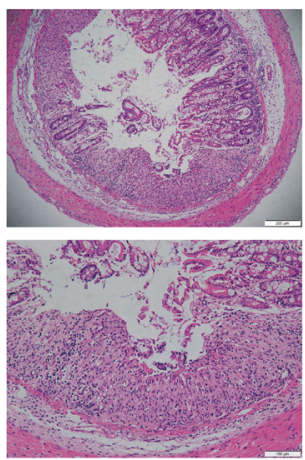

$5 \%$ DSS+PBS

O $5 \%$ DSS+MSC-Exos

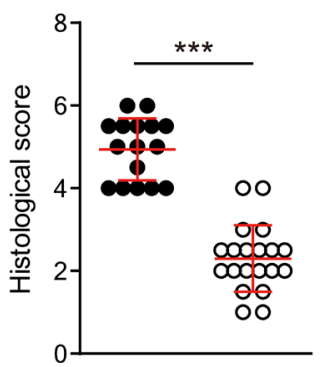

c

- Naive

- $5 \%$ DSS+PBS

O $5 \%$ DSS+MSC-Exos

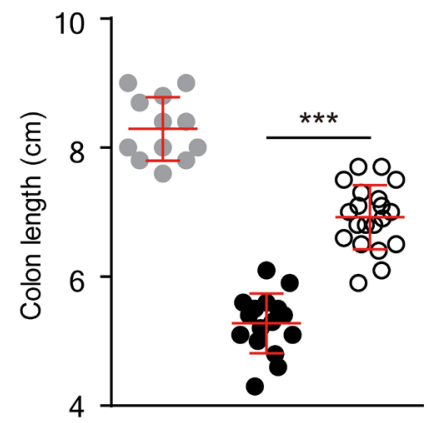

F

- Naive

- $5 \%$ DSS+PBS

O $5 \%$ DSS+MSC-Exos

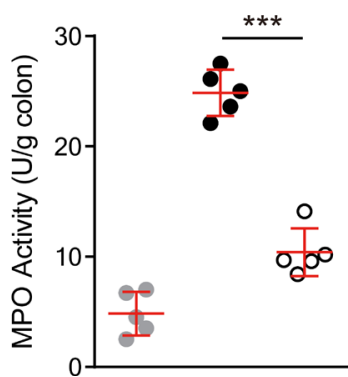

Figure 2. MSC-Exos protect against DSS-induced colitis. Male C57BL/6 mice at 6-8 weeks of age ( $n=12-20$ mice per group from 2 independent but reproducible experiments) were subjected to 5\% DSS in the drinking water for 7 days, and MSC-Exos (200 $\mu \mathrm{g} / \mathrm{mouse}$ ) were infused intravenously on day 2 (arrow in A). DAI scores from body weight loss, stool consistency and blood (A) and body weight (B) were recorded daily. (C) Measurements of colon length from mice on day 7. (D) Histopathological changes in colon tissues analyzed by hematoxylin and eosin (HE) staining on day 7. Original magnification, $\times 100$ (upper), $\times 200$ (lower). (E) Semiquantitative scoring of histopathology performed as described in the Supplemental Methods (supplemental material available online with this article; https://doi.org/10.1172/jci.insight.131273DS1). (F) Neutrophil infiltration determined by measuring colonic MPO activity on day 7. The number of animals studied is shown in each figure. ${ }^{* *} P \leq 0.001$, by Mann-Whitney $U$ test (A and $\left.\mathbf{E}\right)$ or 1 -way ANOVA (B, C, and $\left.\mathbf{F}\right)$.

Effect of MSC-Exos on mucosal inflammation and intestinal barrier integrity. Dysregulation of inflammatory mediators has been well recognized in murine experimental colitis and in patients with IBD (22). Thus, we determined the in vivo effect of MSC-Exos on the production of inflammatory cytokines mechanistically associated with IBD. Compared with untreated DSS-colitic mice, MSC-Exos administration decreased expression of several proinflammatory cytokines, including IFN- $\gamma$, IL-1 $\beta$, IL- 6 , and TNF- $\alpha$, whereas expression of the antiinflammatory cytokine IL-10 was significantly increased (Figure 4, A-E).

Because it is a salient feature of $\operatorname{IBD}(23,24)$, we further investigated the effects of MSC-Exos on intestinal barrier dysfunction. DSS administration increased the serum levels of FITC-dextran, whereas MSC-Exos treatment significantly reduced the DSS-induced increase of intestinal permeability, as shown by significantly decreased levels of FITC-dextran (Figure 4F). An abnormal intestinal barrier could result in increased infiltration of intestinal microbes into intestinal lamina propria (23). Indeed, DSS challenge increased the invasion of colonic tissues by microbes, and this was significantly reduced in mice treated with MSC-Exos (Figure $4 G)$. In accordance with that, DSS administration decreased the mRNA levels of antimicrobial peptides in colons, including lysozyme 1 ( $L y z 1$ ); defensin, alpha, 20 (Defa20); defensin, alpha, 29 (Defa29); and angiogenin, ribonuclease A family, member 4 (Ang4), whereas MSC-Exos treatment reversed these trends (Figure $4 \mathrm{H}$ ). Together, these data indicated that MSC-Exos contribute to the maintenance of gut homeostasis.

The anticolitic benefit of MSC-Exos is macrophagedependent. Subsequently, to elucidate the cell type targeted by implanted MSC-Exos in colitis, we intravenously infused PKH26-labeled MSC-Exos into DSS-colitic mice. 
A

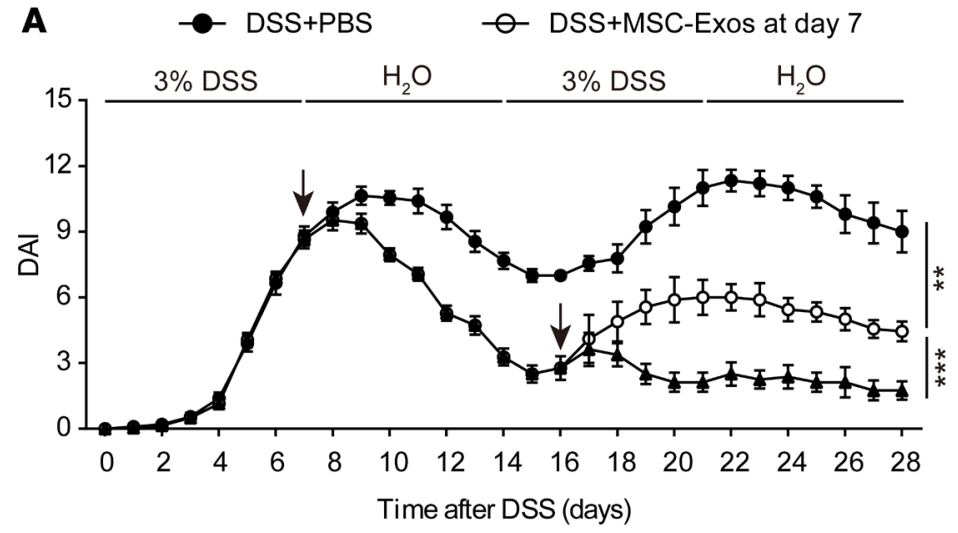

B
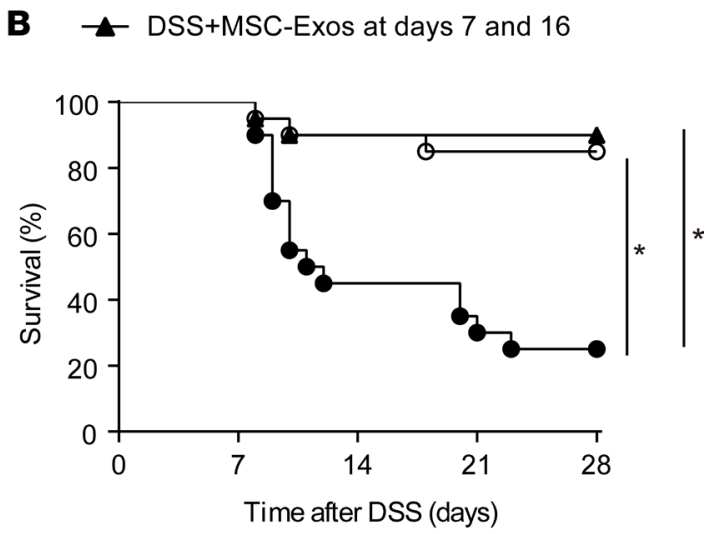

D
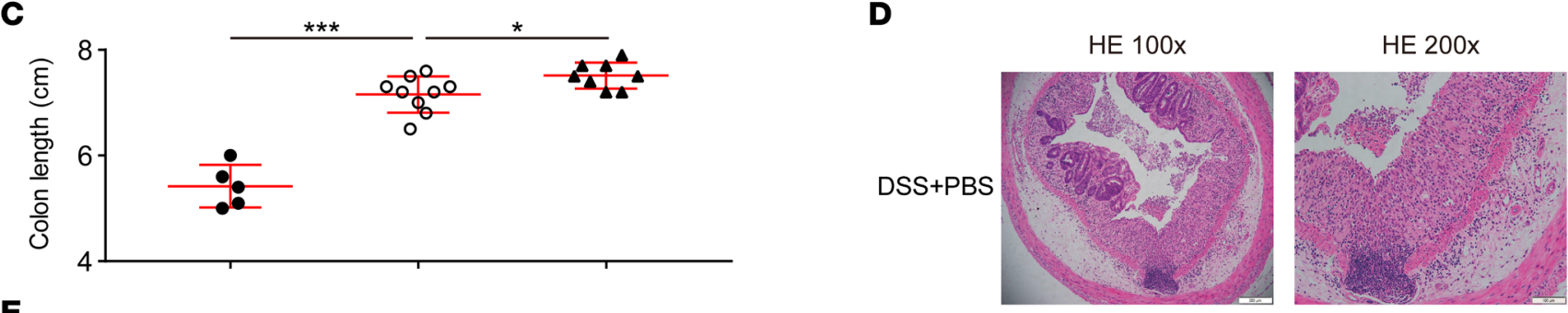

E
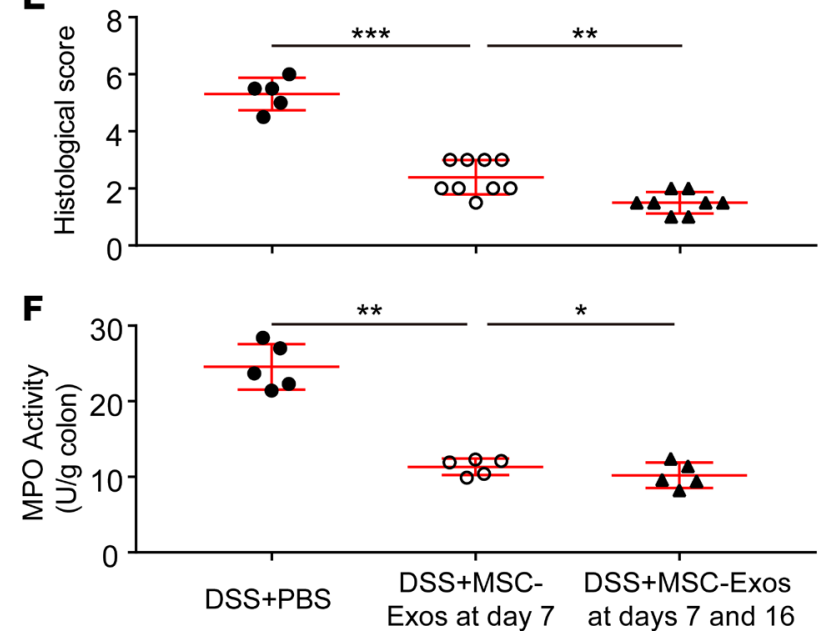

DSS+MSC-Exos at day 7
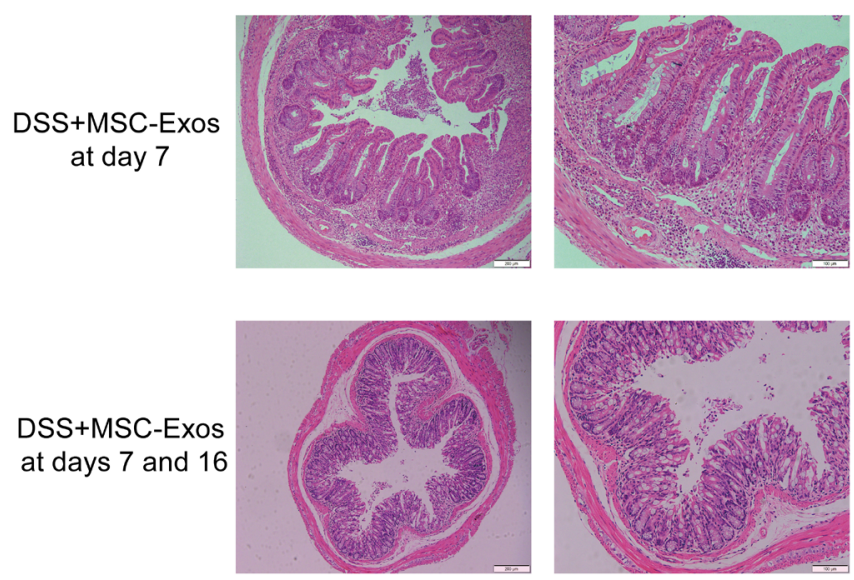

Figure 3. Anticolitic benefit of MSC-Exos in DSS-induced chronic and recurrent colitis. Male C57BL/6 mice at 6-8 weeks of age ( $n=20$ mice per group) were subjected to 3\% DSS in the drinking water in a cyclic manner. Each cycle consisted of 7 days of DSS followed by a 7-day phase without DSS supplementation. MSC-Exos ( $200 \mu \mathrm{g} /$ mouse) were infused intravenously on day 7 or on days 7 and 16 (arrows in A). DAI (A) and mortality (B) were recorded. (C) Measurements of colon lengths. (D) Histopathological changes. Original magnification, $\times 100$ (left), $\times 200$ (right). (E) Semiquantitative scoring of histopathology. (F) Neutrophil infiltration determined by measuring colonic MPO activity on day 28 . The number of animals studied is shown in each figure. ${ }^{*} P \leq$ $0.05,{ }^{* *} P \leq 0.01$, and ${ }^{* * *} P \leq 0.001$, by Kruskal-Wallis test $(\mathbf{A}, \mathbf{E}$, and $\mathbf{F})$, log-rank test (B), or 1-way ANOVA (C).

By analyzing the frequency of exosome-positive cells $\left(\mathrm{PKH} 26^{+}\right)$in a million cells from each colon, we found that MSC-Exos were recruited by the inflamed colon but not by noninflamed intestine (Figure 5, $\mathrm{A}$ and $\mathrm{B}$ ). In addition, about $50 \%$ of $\mathrm{PKH} 26^{+}$cells were $\mathrm{F} 4 / 80^{+} \mathrm{CD} 11 \mathrm{~b}^{+}$as analyzed by flow cytometry, a phenotype consistent with colonic macrophages (Figure 5, A and C). In contrast, labeled MSC-Exos were not efficiently engulfed by colonic macrophages in DSS-untreated mice (Figure 5, A-C). Together, these data suggest that macrophages are the predominant cell type taking up MSC-Exos in colitis and are probably responsible for the anticolitic benefit of MSC-Exos. To substantiate this supposition, we deleted colonic macrophages in mice with intraperitoneal injection of clodronate liposomes (Clod-lipos) (PBS liposomes [PBS-lipos] as negative controls). As anticipated, administration of Clod-lipos effectively deleted colonic macrophages (Supplemental Figure 3, A and B). MSC-Exos were no longer effective in DSS-colitic mice lacking macrophages, providing no significant improvement in DAI, colon length, 

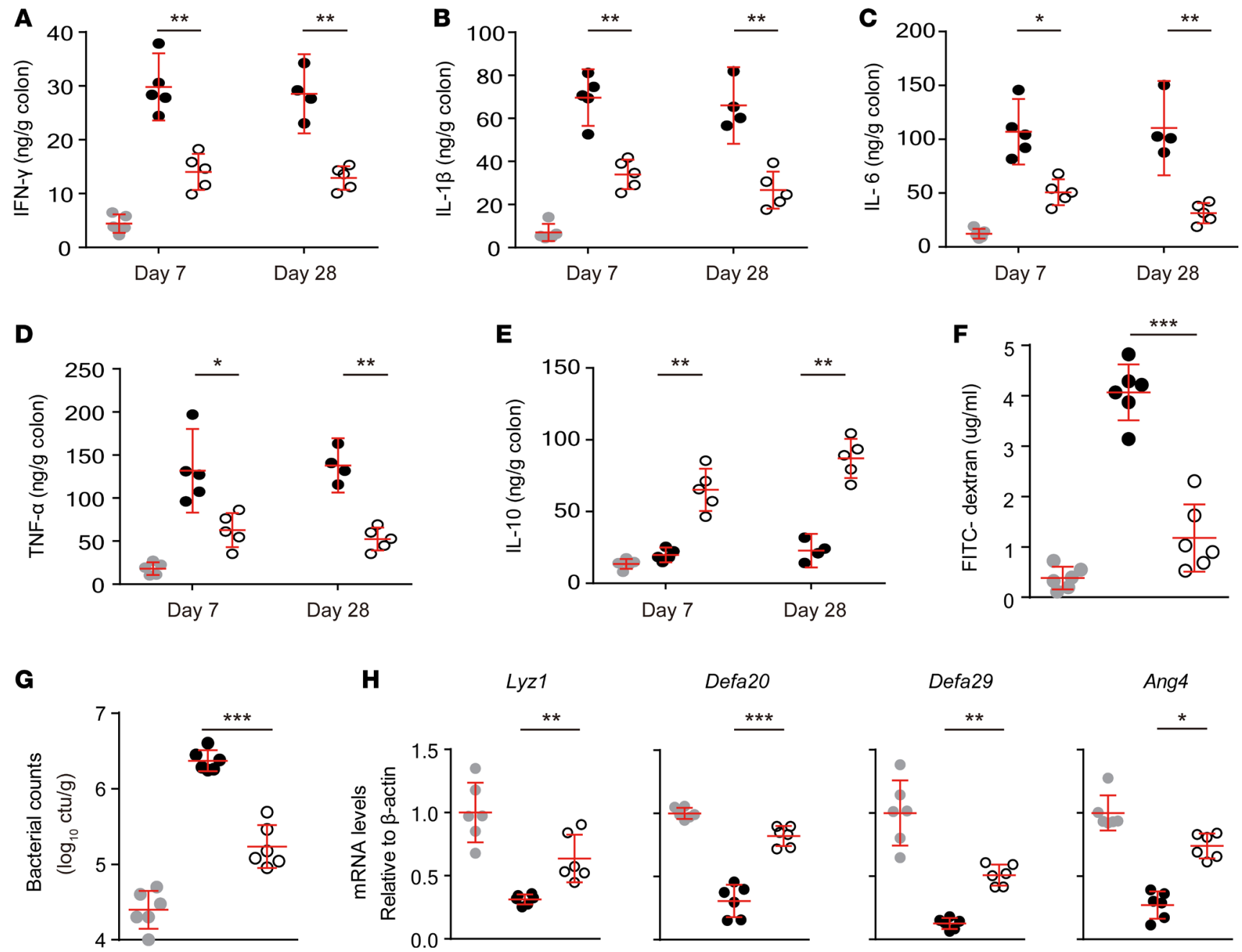

Figure 4. MSC-Exos reduce mucosal inflammatory responses and contribute to the maintenance of intestinal barrier integrity. (A-E) ELISAs for IFN- $\gamma$, IL-1 $\beta$, IL-6, TNF- $\alpha$, and IL-10 in colonic tissues ( $n=5$ mice per group). For F-H, $5 \%$ DSS-colitic mice were killed on day 7 for next detections. (F) FITC-dextran levels in serum 4 hours after oral gavage with FITC-dextran ( $50 \mathrm{mg} / 100 \mathrm{~g}$ body weight) ( $n=6$ mice per group). (C) Bacterial counts in colons from mice with or without MSC-Exos treatment ( $n=6$ mice per group). (H) Quantitative real-time PCR showing expression of antimicrobial peptides in colon samples, including Lyz1, Defa20, Defa29, and Ang4, with Actb as a housekeeping gene ( $n=6$ mice per group). ${ }^{*} P \leq 0.05,{ }^{*} P \leq 0.01,{ }^{*}$ and ${ }^{* *} P \leq 0.001$, by 2 -tailed Student's $t$ test (A-E) or 1-way ANOVA (F-H).

histopathological examination, and colonic MPO activity (Figure 5, D-G). Taken together, these findings indicate that colonic macrophages are essential for the anticolitic benefit of MSC-Exos treatment.

MSC-Exos ameliorate mucosal inflammation by remodeling macrophage phenotypes. Colonic macrophagemediated mucosal inflammatory responses play a central role in the onset and severity of IBD. To delineate the mechanism of the anticolitic benefit of MSC-Exos, we investigated the in vivo effects of MSC-Exos on colonic macrophages. Despite no significant change in the percentage of $\mathrm{F} 4 / 80^{+} \mathrm{CD} 11 \mathrm{~b}^{+}$cells in the lamina propria of colons, the expression levels of CD206 and arginase-1 in colonic macrophages were significantly elevated upon MSC-Exos treatment (Figure 6, A-D). As anticipated, the colonic expression of markers indicative of M2 macrophages was also significantly upregulated upon transfer of MSC-Exos (Supplemental Figure 4, A and B). More excitingly, macrophages isolated from mice treated with MSC-Exos produced less IL-1 $\beta$, IL- 6 , and TNF- $\alpha$ but more IL-10 on ex vivo culture (Figure $6 \mathrm{E}$ ), revealing that the reduction of mucosal inflammatory responses upon MSC-Exos treatment might be a consequence of direct action on colonic macrophages. Importantly, macrophages from colitic colons acquired obvious resistance to LPS restimulation when prepared from mice treated with MSC-Exos versus untreated mice (Figure 6E). Altogether, these data suggest that MSC-Exos attenuate mucosal inflammation by polarizing M2b macrophages. 
A
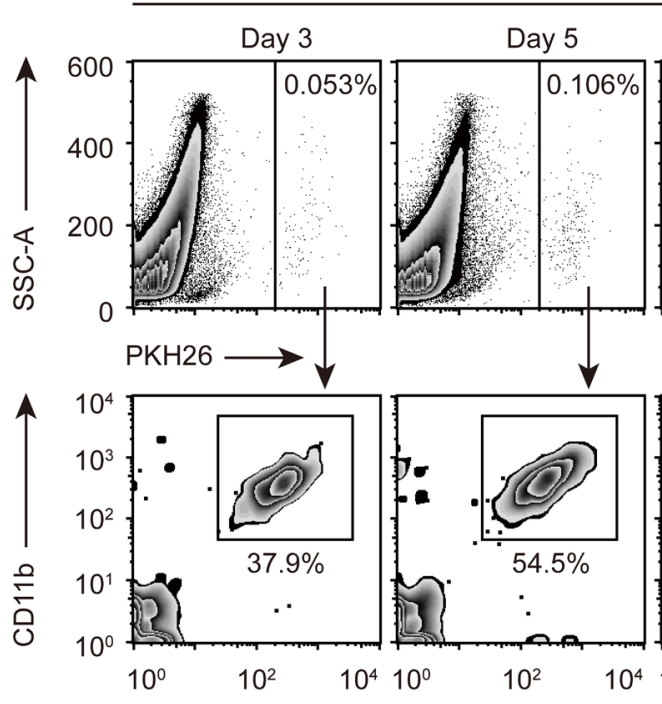

F4/80
$5 \%$ DSS-treated mice

B

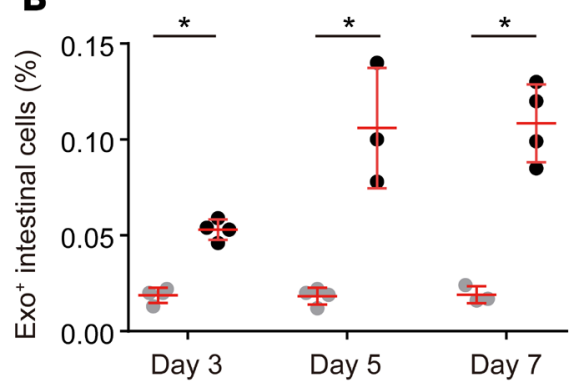

- $3 \%$ DSS+PBS-lipo+PBS

D $-3 \%$ DSS+PBS-lipo+MSC-Exos

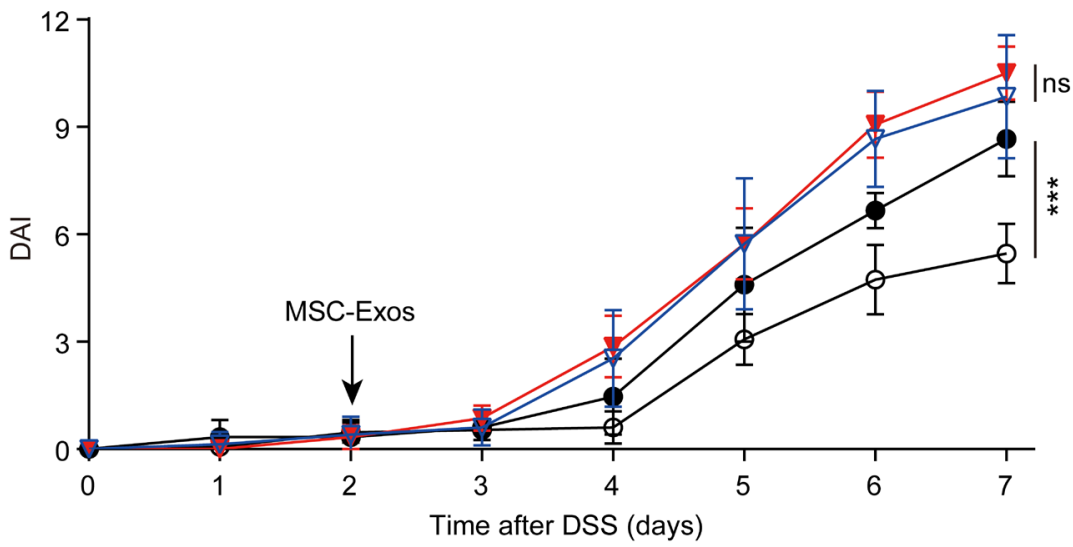

DSS-untreated mice

C

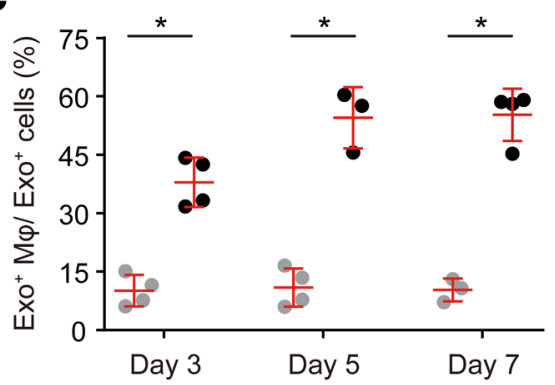

$\neg$ 3\% DSS+Clod-lipo+PBS

$\nabla$ 3\% DSS+Clod-lipo+MSC-Exos

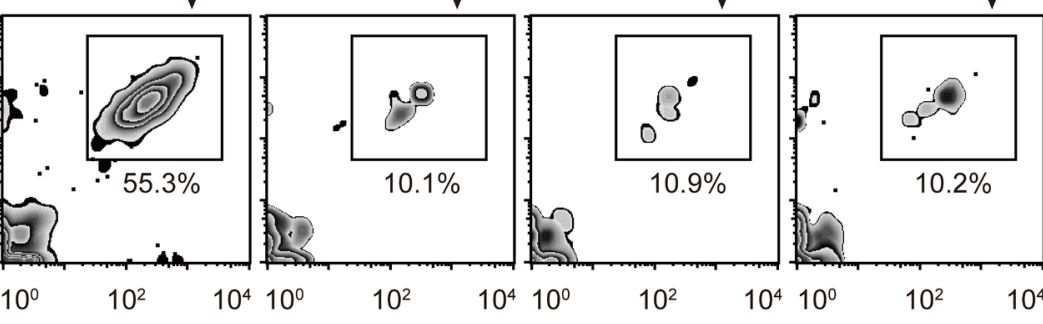

$3 \%$ DSS+PBS

O $3 \%$ DSS+MSC-Exos

E

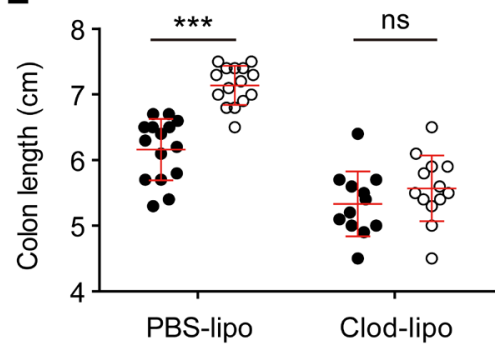

$\mathbf{F}$

DSS-untreated mice
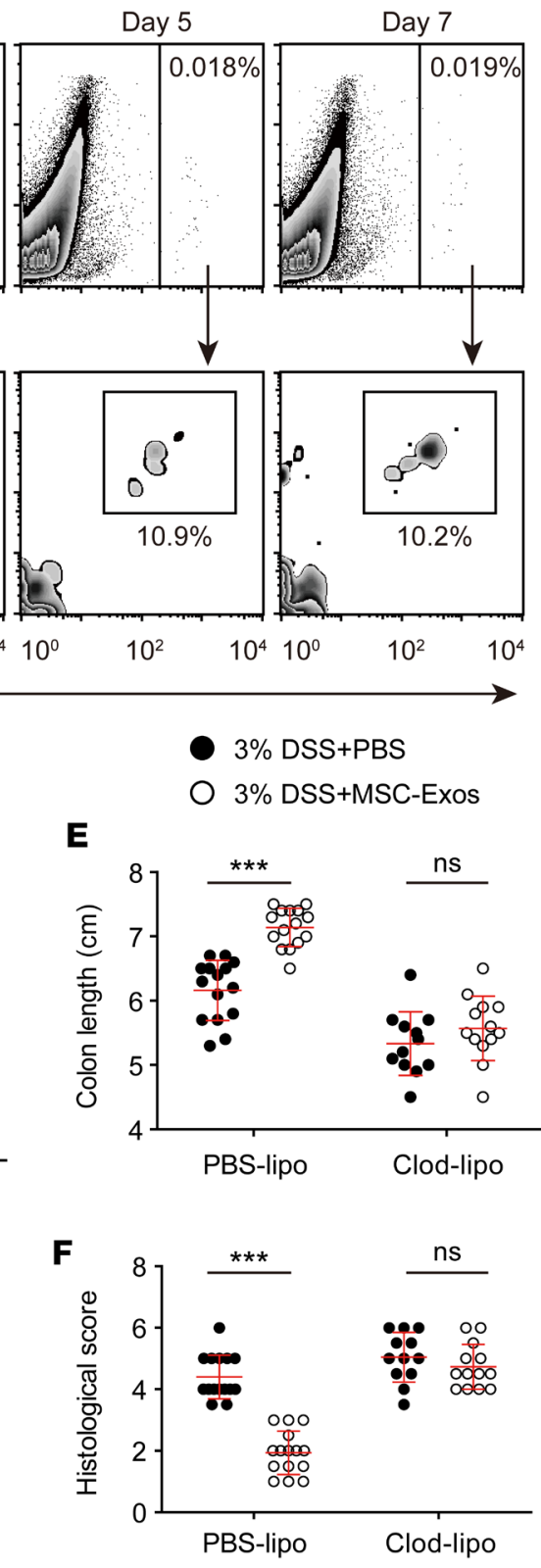

G

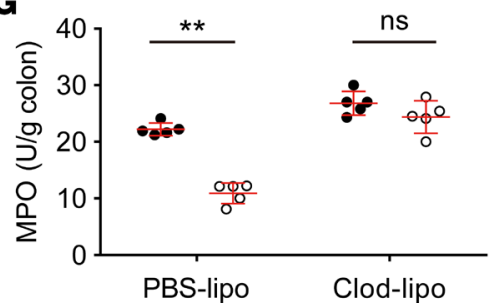

Figure 5. The anticolitic benefit of MSC-Exos in DSS-colitic mice is macrophage dependent. PKH26-labeled MSC-Exos (200 $\mu \mathrm{g}$ per mouse) were intravenously administrated to mice on day 2 during the 7-day 5\% DSS administration. Mice were sacrificed on days 3, 5, and 7 for tracking analysis. (A) Upper: The frequency of $\mathrm{PKH}_{26}{ }^{+}$intestinal cells dissociated from DSS-treated or untreated mice (1 million cells were detected in each colon). Numerical values denote the mean percentage of exosome-positive ( $\mathrm{PKH}_{2} \mathrm{6}^{+}$) intestinal cells. Lower: Flow cytometric profiles of F4/80 and CD11b expression in PKH26+ cells. Numerical values denote the mean percentage of $\mathrm{PKH}_{26} 6^{+}$cells expressing CD11b and F4/80. (B) The quantification of PKH26+ cells. (C) The quantification of PKH26 ${ }^{+}$ cells expressing F4/80 and CD11b. $n=3-4$ mice per group for A-C. For D-G, mice received Clod-lipos or PBS-lipos according to the schematic flowchart (see Supplemental Figure 3A), and MSC-Exos (200 $\mu$ g per mouse) were infused intravenously on day 2 (arrow in D). Colitis was assessed by DAI (D) daily. On day 7 , mice were sacrificed, and colon lengths (E), histopathological scores (F), and colonic MPO activity (G) were determined. $n=12-15$ mice per group for $\mathbf{D}-\mathbf{F}$ and $n=5$ for $\mathbf{C} .{ }^{*} P \leq 0.05,{ }^{* *} P \leq 0.01,{ }^{* *} P \leq 0.001$, and ns indicates $P>0.05$, by 2 -tailed Student's $t$ test $(\mathbf{B}, \mathbf{C}, \mathbf{E}$, and $\mathbf{G})$ or Mann-Whitney $U$ test $(\mathbf{D}$ and $\mathbf{F})$. 
The effect of MSC-Exos on intestinal fibrosis was also analyzed. ELISAs (Supplemental Figure 4C) and reverse transcription PCR gels (Supplemental Figure 4D) revealed no significant differences in the levels of collagen in MSC-Exos-treated mice compared to controls. As shown by Masson's trichrome staining, DSS administration led to increased collagen in the colons of chronic DSS-colitic mice, whereas MSC-Exos treatment did not result in increased colonic collagen (Supplemental Figure 4E). Collectively, these data indicate that MSC-Exos treatment does not favor intestinal fibrosis.

Macrophage-derived IL-10 is key for the beneficial effect of MSC-Exos. Because MSC-Exos treatment increased colonic expression of the immunosuppressive cytokine IL-10 (Figure 4E), we asked whether IL-10 is important for the anticolitic benefit of MSC-Exos in DSS-colitic mice. Administration of an antibody against IL-10 in mice treated with MSC-Exos and 5\% DSS yielded intermediate DAI and colonic MPO activity compared with time-matched mice treated with DSS and MSC-Exos plus DSS (Figure 7A). Therefore, IL-10 is an important component of the anticolitic benefit of MSC-Exos. We next set out to understand the source of IL-10 elevation in colons upon MSC-Exos treatment. It is unlikely that the injected MSC-Exos were the source of the elevated IL-10 because the protein cargo contained in MSC-Exos did not include IL-10 as determined by Western blot and ELISA (data not shown). Large amounts of IL-10 are produced mainly by type 2 helper $\mathrm{T}$ (Th2) cells, T regulatory cells, and macrophages $(25,26)$. To assess the involvement of $\mathrm{T}$ cells in elevating IL-10, we isolated $\mathrm{CD} 4^{+} \mathrm{T}$ cells from the mesenteric lymph nodes (MLNs) of DSS-colitic mice. On ex vivo culture, CD4 ${ }^{+} \mathrm{T}$ cells with MSC-Exos treatment did not upregulate IL-10 expression, even after restimulation with phytohemagglutinin (PHA) (Figure 7B). In addition, the Th1 and Th2 cell profile of $\mathrm{CD}^{+} \mathrm{T}$ cells was not significantly influenced upon MSC-Exos treatment (Figure 7C and Supplemental Figure 4F). Macrophage depletion impaired the anticolitic effect of MSCExos, supporting the notion that macrophages might be the source of the elevated IL-10 in colons upon MSC-Exos treatment. Further supporting this hypothesis, $\mathrm{F} 4 / 80^{+}$macrophages isolated from mice treated with MSC-Exos produced more IL-10 on ex vivo culture (Figure 6E). Together, these data suggest that the elevated IL-10 in colons upon MSC-Exos treatment is at least partially derived from colonic macrophages and is probably not associated with Th2 or T regulatory cells.

To further support the involvement of macrophage-derived IL-10 in the beneficial effects of MSC-Exos, we analyzed the regulatory potential of macrophages educated by MSC-Exos on CD4 ${ }^{+} \mathrm{T}$ cells. In contrast to control macrophages, those educated by MSC-Exos and IL-4 significantly inhibited the proliferation of $\mathrm{CD}^{+} \mathrm{T}$ cells at ratios as low as 1:10 (Figure 7, E and F) and significantly suppressed the production of the inflammatory cytokines TNF- $\alpha$ and IFN- $\gamma$ (Figure 7, G and H). The IL-10 blockade partially reversed the inhibitory activity of macrophages educated by MSC-Exos on activation of CD4 ${ }^{+} \mathrm{T}$ cells (Figure 7, D-H).

MSC-Exos inhibit the inflammatory response of macrophages via transporting metallothionein-2. To understand the molecular basis mediating the effects of MSC-Exos, we performed proteomic analysis to analyze the protein expression profiles in MSC-Exos and the corresponding supernatants from their parent MSCs (PRIDE Archive, Project PXD015386). We identified 1315 protein groups, among which 992 proteins were quantified. All the identified proteins were biologically interpreted, and their involved biological processes were explored. MSC-Exos were enriched in proteins involved in regulating multiple biological processes related to the anticolitic benefit of MSC-Exos, such as immune modulation, cytokine secretion, NF- $\mathrm{kB}$ signaling pathway, acute inflammatory response regulation, and collagen catabolic process (Figure 8A). We selected 389 differentially expressed proteins by volcano plot filtering between MSC-Exos and the corresponding supernatants (fold change $\geq 2$ and $P \leq 0.05$ ) (Figure $8 \mathrm{~B}$ ). Among them, metallothionein-2, a high conservative protein between Homo sapiens and Mus musculus, was the most highly upregulated by more than 9-fold in MSC-Exos compared with the supernatants (Figure 8B). ELISAs confirmed the significant enrichment of metallothionein-2 in MSC-Exos (Figure 8C), and metallothionein-2 appeared to be uniquely upregulated in MSC-Exos (Supplemental Figure 5A). In addition, the presence of metallothionein-2 in exosomes was necessary to have uptake into macrophages (Supplemental Figure 5B), which at least partly explained why free metallothionein-2 had no activity on the polarization of macrophages (Supplemental Figure 5, C and D).

To investigate the role of metallothionein-2 in the beneficial effect of MSC-Exos in macrophages, 3 siRNAs ( $\left(\right.$ iMT2 $\mathrm{A}^{\# 1}$, siMT2A $\mathrm{A}^{\# 2}$, and siMT2A ${ }^{\# 3}$ ) were used to knock down metallothionein-2 expression in MSC-Exos. The inhibitory efficiency of these siRNAs was tested by ELISAs. Results from ELISAs showed the downregulation of metallothionein-2 in MSC-Exos from $M T 2 A$-silenced MSCs (MSC-Exos ${ }^{\text {siMT2A\#2 }}$ ) (Figure 8C). As expected, the reduction of metallothionein-2 in MSC-Exos interfered with the antiinflammatory effects of MSC-Exos both in vitro (Figure 8D) and in vivo (Supplemental Figure 6, A-C). 
A

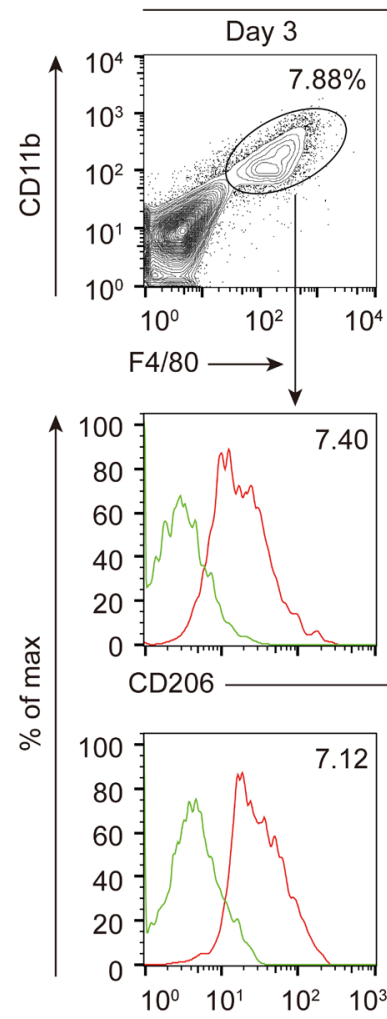

5\% DSS plus PBS treated mice
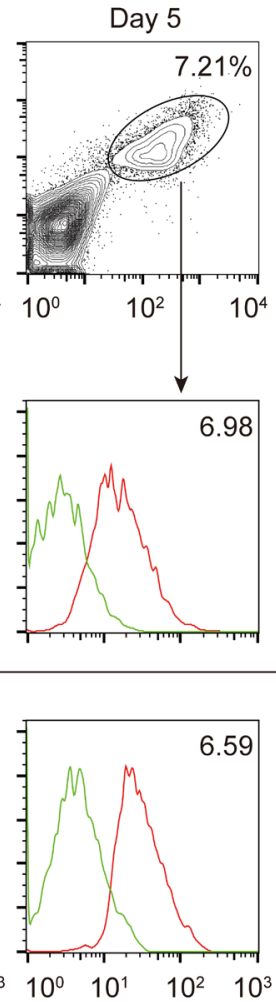
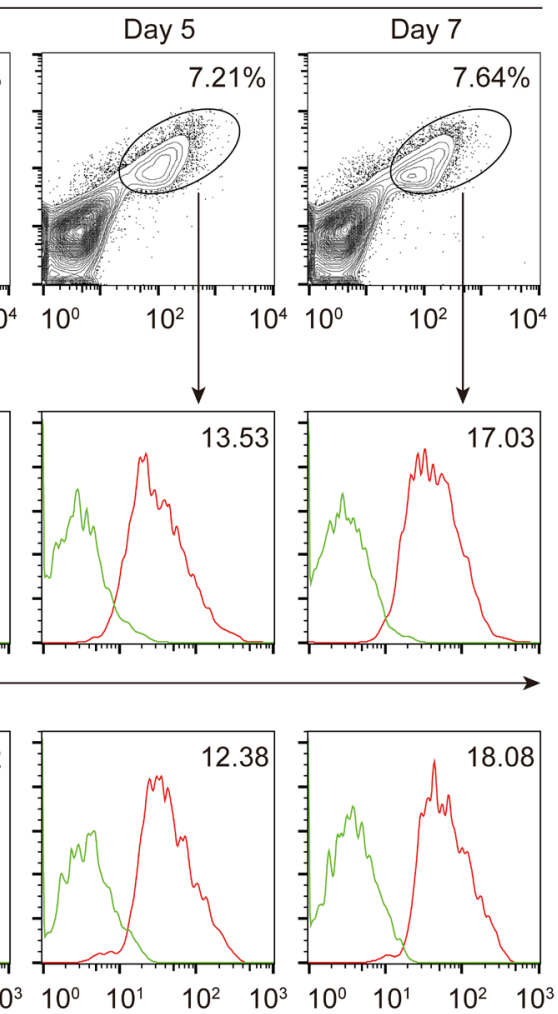

arginase-1
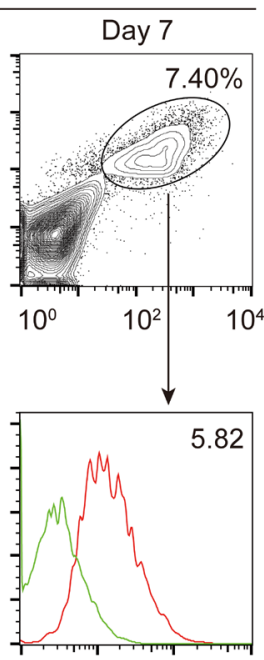

82
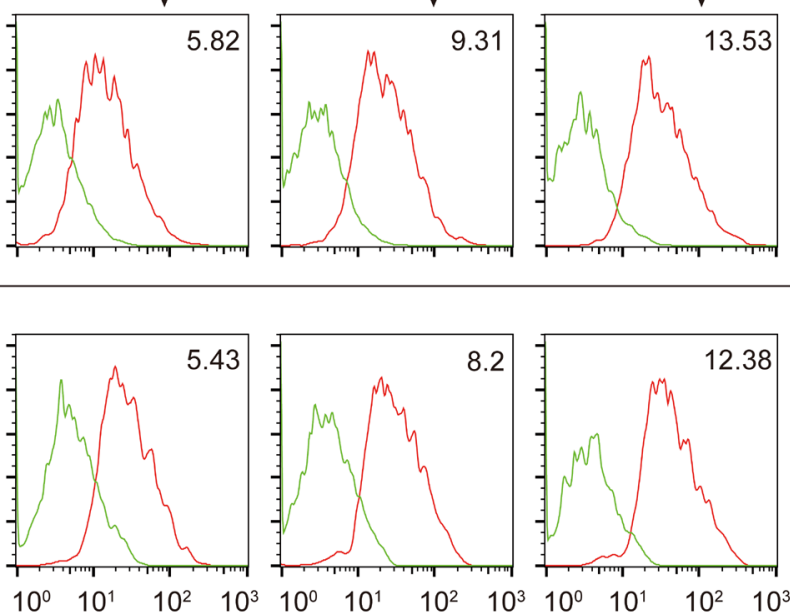

5\% DSS plus MSC-Exos treated mice

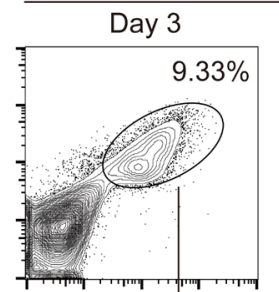

$5 \%$ DSS+PBS O $5 \%$ DSS+MSC-Exos
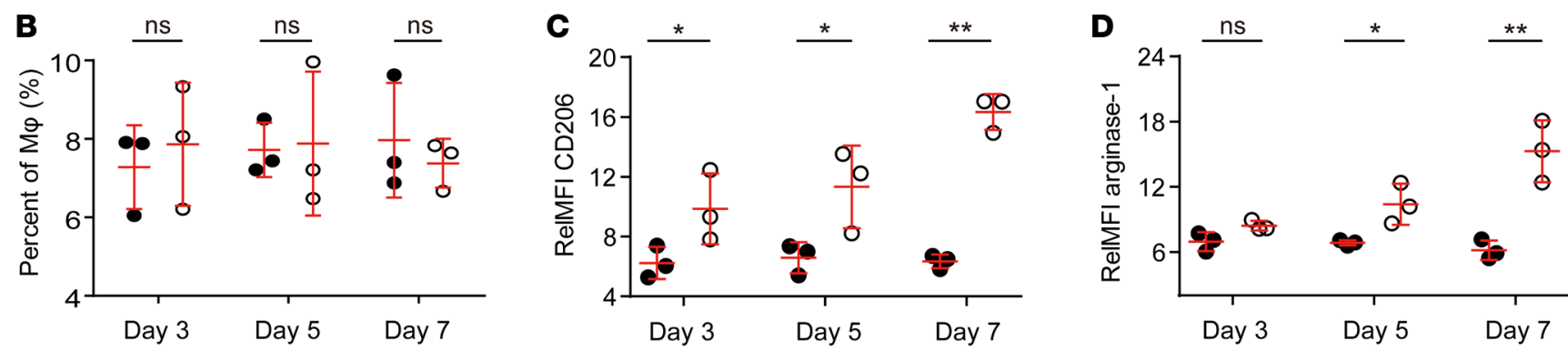

\section{E}

$\mathrm{IL}-1 \beta(\mathrm{pg} / \mathrm{ml})$
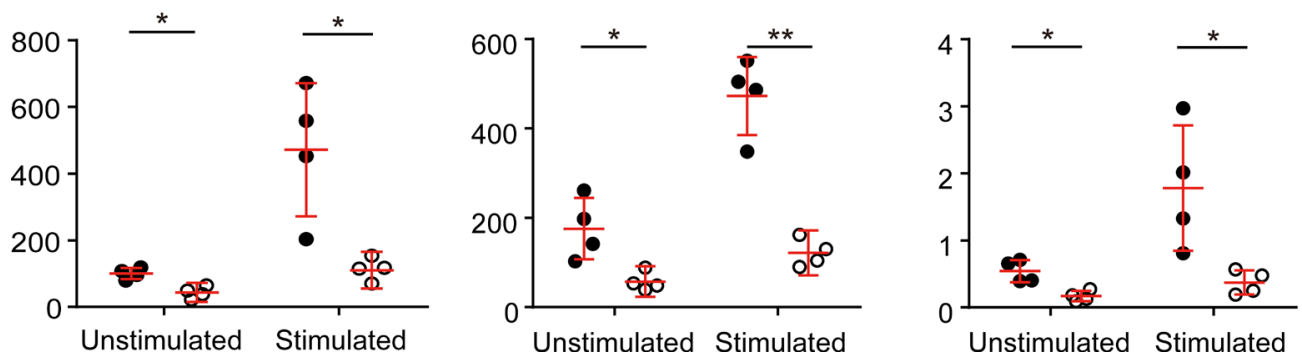

$\mathrm{IL}-10(\mathrm{ng} / \mathrm{ml})$

Figure 6. MSC-Exos reduce mucosal inflammation by polarizing M2b macrophages. (A) Upper: Percentage of cells expressing F4/80+CD11b+ in lamina pro-

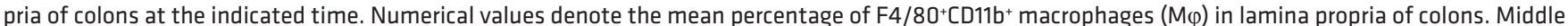
and lower: Expression of CD206 and arginase-1 in macrophages. Numerical values denote the relative mean fluorescence intensity (RelMFI) normalized to fluorescence minus 1 control. Quantification of macrophages (B) and of macrophages expressing CD206 (C) and arginase-1 (D) in the lamina propria of colons. $n=3$ mice/group in A-D. (E) The cytokine contents in culture supernatants of F4/80+ macrophages isolated at day 7 from $5 \%$ DSS-colitic mice with or without MSC-Exos treatment on ex vivo 24 -hour culture with or without $100 \mathrm{ng} / \mathrm{mL}$ LPS restimulation ( $n=4$ mice/group). ${ }^{*} P \leq 0.05,{ }^{*} P \leq 0.01$, and $\mathrm{ns}$ indicates $P>0.05$, by 2 -tailed Student's $t$ test (B-E). 
A

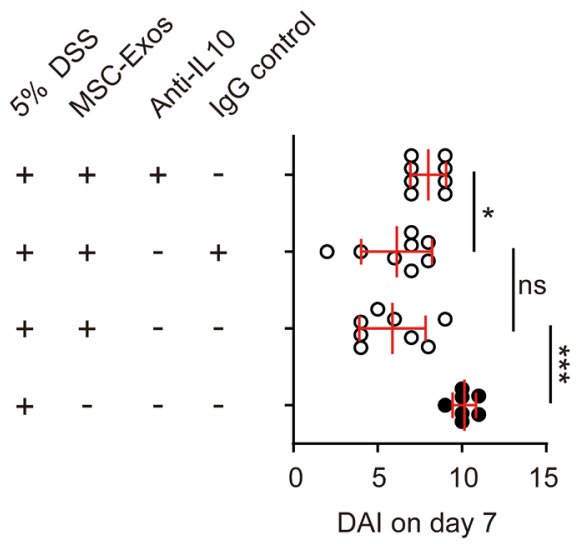

B

- Control O MSC-Exos

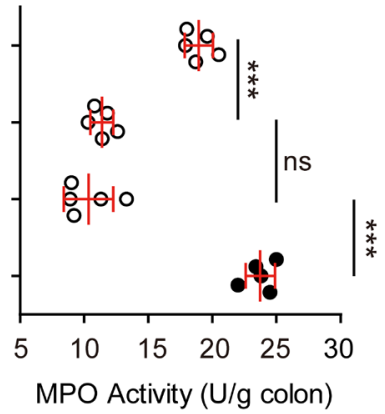

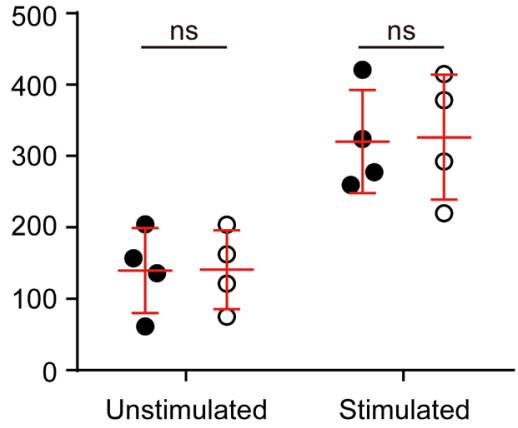

C

Control O MSC-Exos
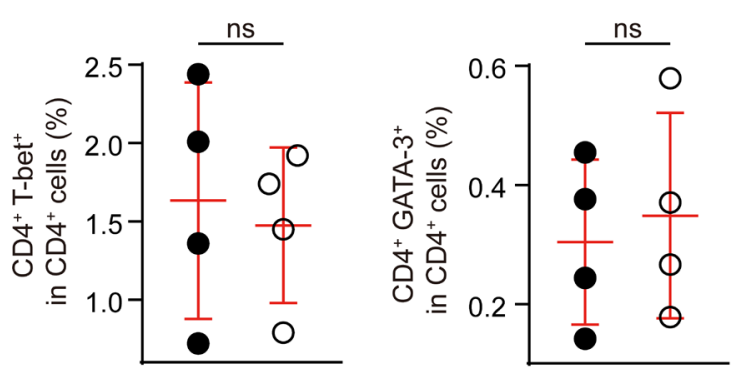

D

\begin{tabular}{lllllll}
\hline Groups: CD4 ${ }^{+}$T cells coculture with & a & b & c & d & e & f \\
\hline Control macrophages & - & + & - & - & - & - \\
MSC-Exos treated macrophages & - & - & + & - & + & + \\
IL-4 treated macrophages & - & - & - & + & - & - \\
IgG control $(10 \mu \mathrm{g} / \mathrm{ml})$ & - & - & - & - & + & - \\
Anti-IL-10 antibodies $(10 \mu \mathrm{g} / \mathrm{ml})$ & - & - & - & - & - & + \\
\hline
\end{tabular}
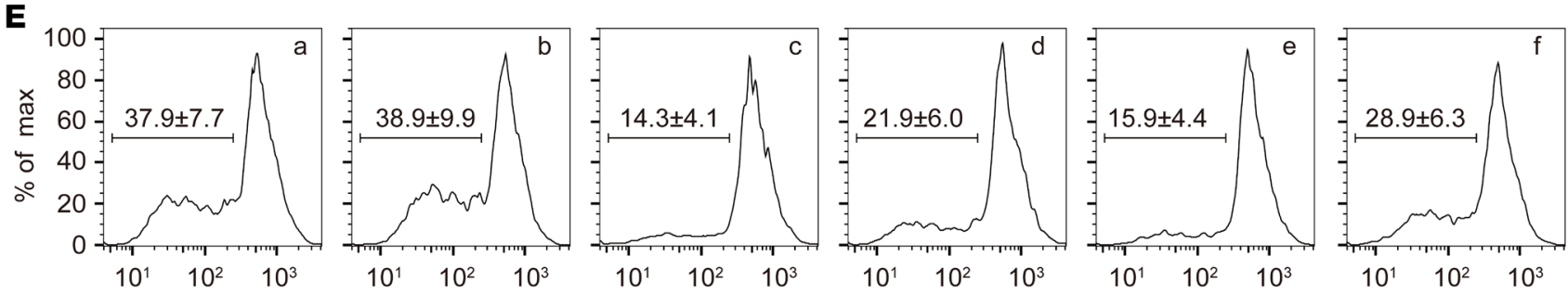

$\mathbf{F}$

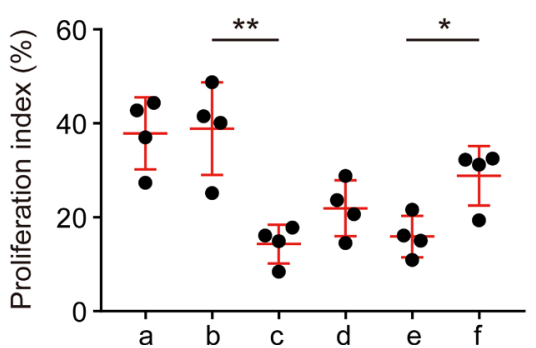

G

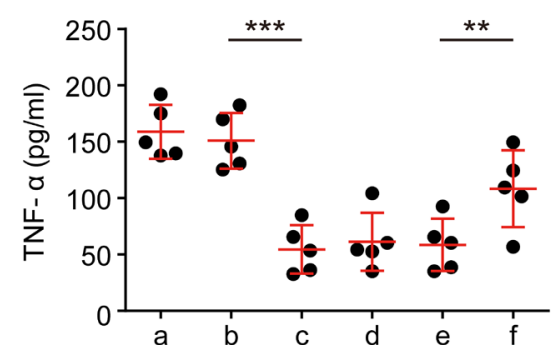

H

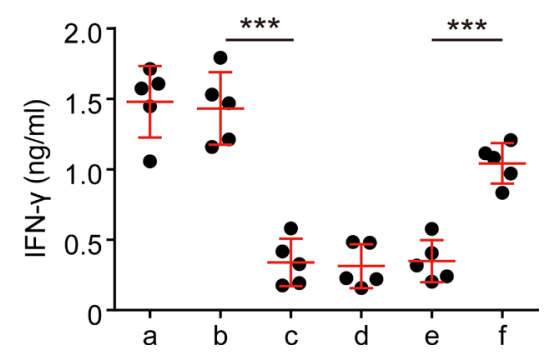

Figure 7. The anticolitic benefit of MSC-Exos is partially dependent on macrophage-derived IL-10. (A) Intermediate DAI and colonic MPO activity in mice receiving MSC-Exos plus an antibody against IL-10 compared to DSS- and MSC-Exos plus DSS-treated mice ( $n=8$ mice per group). (B) CD4 ${ }^{+}$ cells isolated from MLNs of $5 \%$ DSS-colitic mice at day 7 were ex vivo cultured with or without MSC-Exos (30 $\mu \mathrm{g} / \mathrm{mL})$ for 2 days. After restimulation with or without $5 \mu \mathrm{g} / \mathrm{mL}$ PHA for 24 hours, the cytokine IL-10 was determined in culture supernatants ( $n=4$ mice per group). (C) CD4 ${ }^{+}$T cells isolated from human peripheral blood mononuclear cells were treated with or without MSC-Exos $(30 \mu \mathrm{g} / \mathrm{mL})$ for 2 days, and Th1 and Th2 flow cytometric profiles were determined ( $n=4$ independent experiments). (D-H) Human peripheral blood monocyte-derived macrophages were cultured with MSCExos $(30 \mu \mathrm{g} / \mathrm{mL})$ or IL-4 $(20 \mathrm{ng} / \mathrm{mL})$ for 2 days. Cells were harvested and cocultured with human peripheral blood-derived CD4 ${ }^{+} \mathrm{T}$ cells at a ratio of 1:10 stimulated with PHA $(5 \mu \mathrm{g} / \mathrm{mL})$ using a transwell system in the presence or absence of anti-IL-10 antibodies $(10 \mu \mathrm{g} / \mathrm{mL})$ or an isotype-matched IgC control. (D) The study design for the coculture experiments shown in E-H. (E) Proliferation of 5,6-carboxyfluorescein diacetatesuccinimidyl esterlabeled (CFSE-labeled) CD4 ${ }^{+}$T cells assessed after 3-day coculture by flow cytometry. Numbers denote the percentage of cells undergoing at least 1 cellular division (mean \pm SD, $n=4$ independent experiments). (F) Quantification of proliferation of CFSE-labeled CD4 ${ }^{+} T$ cells in E. (G and $\left.\mathbf{H}\right)$ After 3-day coculture, CD4 ${ }^{+}$T cells were harvested and restimulated with PHA $(5 \mu \mathrm{g} / \mathrm{mL})$ for 24 hours, and then TNF- $\alpha$ and IFN- $\gamma$ contents were measured in supernatants by ELISA ( $n=5$ independent experiments). ${ }^{*} P \leq 0.05$, ${ }^{* *} P \leq 0.01$, ${ }^{* *} P \leq 0.001$, and ns indicates $P>0.05$, by Kruskal-Wallis test (A), 2-tailed Student's $t$ test (B and $\mathbf{C})$, or 1-way ANOVA (F, G, and $\mathbf{H})$. 
A

Immune response-regulating signaling pathway

Regulation of innate immune response

Epithelial cell proliferation

Regulation of homeostatic process Negative regulation of immune system process

TNF-mediated signaling pathway

NIK/NF-kappaB signaling

Collagen catabolic process

Cytokine secretion

Regulation of acute inflammatory response

Regulation of macrophage cytokine production

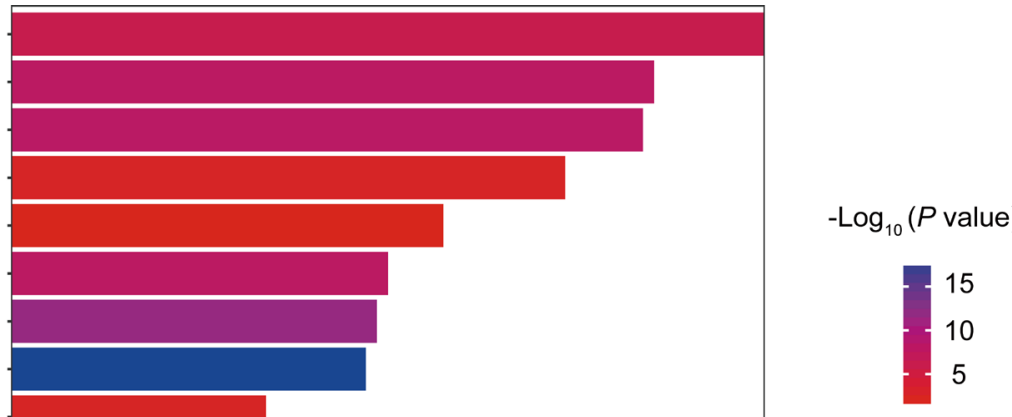

B

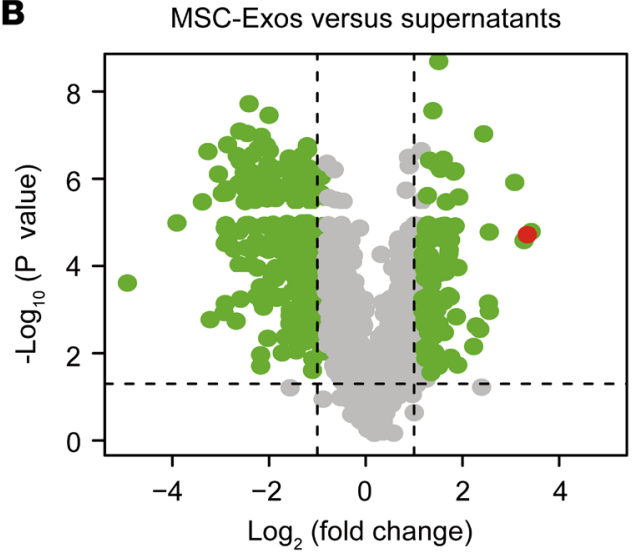

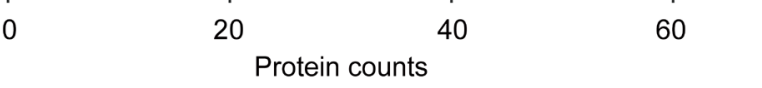
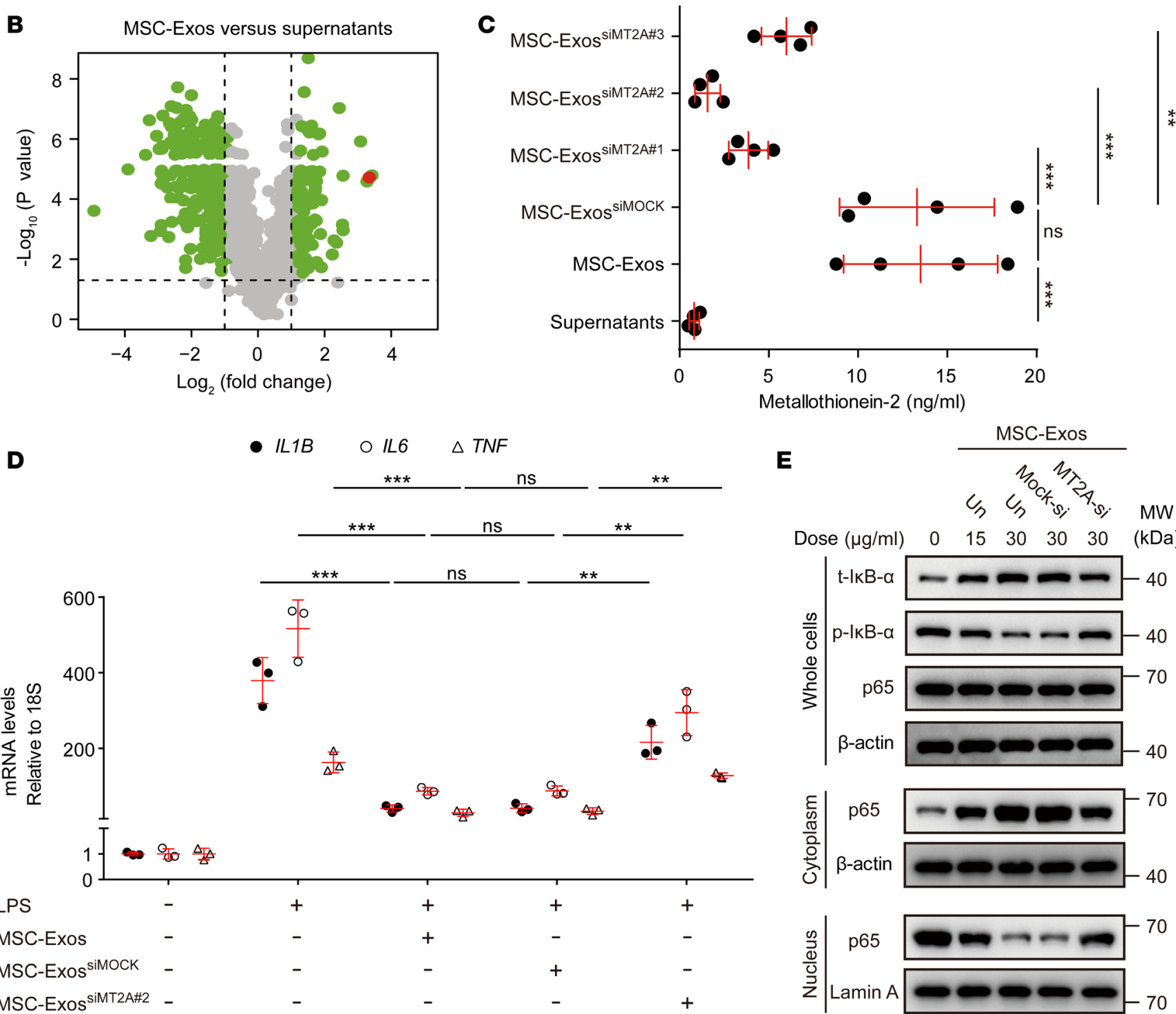

$\mathbf{E}$

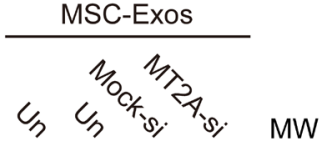

Dose $(\mu \mathrm{g} / \mathrm{ml}) \quad 0 \quad 15 \quad 30 \quad 30 \quad 30 \quad(\mathrm{kDa})$
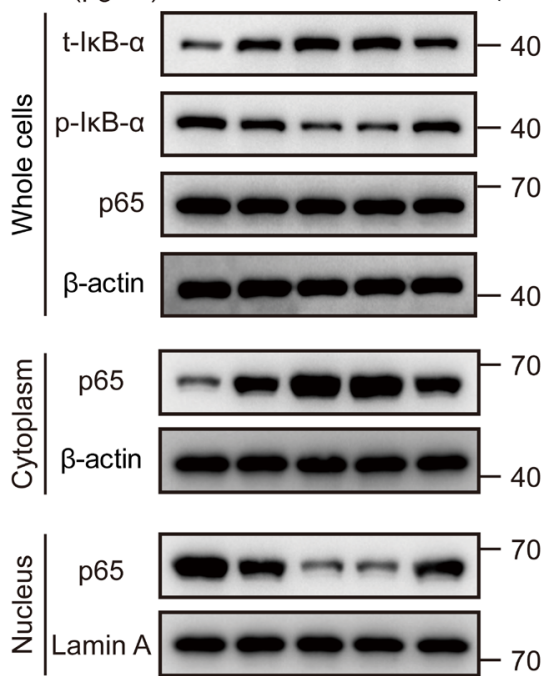

Figure 8. MSC-Exos reduce inflammatory responses in macrophages by transporting metallothionein-2. (A) Biological processes (Gene Ontology terms) related to anticolitic benefit. (B) Volcano plot showing the differential abundance of protein expression in MSC-Exos compared with the corresponding supernatants of exosome depletion. The vertical dotted lines correspond to 2 -fold increase and decrease, respectively, and the horizontal dotted line represents a $P$ value of 0.05 . Metallothionein- 2 is annotated on the volcano plot by the red point. $n=3$ in $\mathbf{A}$ and $\mathbf{B}$. (C) The levels of metallothionein-2. Supernatants of MSCs were depleted of exosomes by ultracentrifugation, and all samples were adjusted to have an equal total protein concentration; then ELISAs were performed. $n=4$ independent experiments. (D) mRNA levels of TNF, IL6, and IL1B in macrophages derived from human 


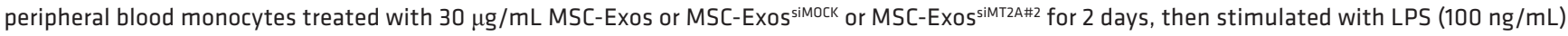
during the last 4 hours, with $18 \mathrm{~S}$ rRNA as a housekeeping gene. $n=3$ independent experiments. (E) Representative immunoblot for phosphorylated $I \kappa B \alpha(p-\mid \kappa B \alpha)$, total $\mid \kappa B \alpha(t-\mid \kappa B \alpha)$, and $p 65$ subunit from whole-cell, nuclear, and cytoplasmic extracts in macrophages that were treated for 2 days, with the indicated dose of MSC-Exos from MSCs that were untransfected (Un), mock transfected (Mock-si), or transfected with MT2A-siRNAs (MT2A-si). Three independent experiments were performed and yielded similar results. $\beta$-actin and Lamin A served as loading controls. ${ }^{*} P \leq 0.01$; ${ }^{* *} P \leq 0.001$; ns indicates $P>0.05$, by Kruskal-Wallis test (C and $\left.\mathbf{D}\right)$.

A previous study revealed that metallothionein-2 could attenuate NF- $\mathrm{B}$ activity (27). Indeed, treatment with MSC-Exos dose-dependently resulted in reduced level of phosphorylated $\mathrm{I} \kappa \mathrm{B} \alpha$ in macrophages but increased total I $\mathrm{B} \alpha \alpha$ level in both protein and mRNA (Figure 8E and Supplemental Figure 6D). A reduction of NF- $\mathrm{BB}$ p65 subunit nuclear translocation was also observed (Figure 8E). Furthermore, knockdown of metallothionein-2 significantly reversed the increase of IkB $\alpha$ mRNA levels induced by MSC-Exos (Supplemental Figure 6D). As expected, the inhibitory effect of MSC-Exos on NF- $\mathrm{BB}$ activation was compromised as well (Figure $8 \mathrm{E}$ ). It has been reported that metallothionein-2 could enhance I $\mathrm{B} \alpha$ transcription via interacting with myeloid zinc finger $1(M Z F 1)$, which directly bound to $\mathrm{I} \kappa \mathrm{B} \alpha$ promoter (27). In agreement with this, MSC-Exos failed to enhance I $\mathrm{B} \alpha$ transcription in macrophages when $M Z F 1$ was knocked down using siRNA (Supplemental Figure 6E), implying a vital role of $M Z F 1$ on the increase of IкB $\alpha$ transcription. Taken together, these findings revealed that metallothionein-2 transmitted by MSC-Exos was likely to suppress $\mathrm{NF}-\kappa \mathrm{B}$ activation via $M Z F 1$ by enhancing $\mathrm{I} \kappa \mathrm{B} \alpha$ transcription and inhibiting the phosphorylation of $\mathrm{I} \kappa \mathrm{B} \alpha$.

\section{Discussion}

Current available medical therapeutic options for IBD have not been able to completely halt the progression of IBD or to change its natural history. Therefore, designing optimal therapeutic strategies has been the subject of intense investigation. We propose a new therapeutic tool for IBD treatment in murine preclinical models using MSC-Exos, pivoting from MSC-based cell transplantation to cell-free therapeutics. Systemic infusion of MSC-Exos at the disease onset was sufficient to reduce the severity of acute colitis, and administration of MSC-Exos in mice significantly ameliorated the severity of chronic colitis. An initial MSC-Exos infusion also prevented the recurrence of the disease after subsequent DSS administration. In addition, the anticolitic effect of MSC-Exos is mouse strain independent. MSC-Exos treatment efficiently ameliorated colitis in both C57BL/6 and BALB/c strains. These results are potentially relevant with respect to disease intervention.

A critical concern is whether the use of human exosomes in animal models, a xenogeneic material systemic administration, would elicit immune rejection. Theoretically, exosomes are non-immune rejective (20). Indeed, when using human exosomes in animal models, numerous studies have revealed significant therapeutic effects in several diseases, and no immune rejection to date has been observed in the xenogeneic system $(11,16,20,28)$. In line with the previous reports $(11,16,20,28)$, no significant immunological rejection was detected when MSC-Exos were administrated to xenogeneic hosts in this study (Supplemental Figure 7, A-D). Additionally, class I and II major histocompatibility complex, key mediators of potential immune rejection, were absent on MSC-Exos as determined by Western blot (data not shown). These findings suggest that the immunosuppressive action of MSC-Exos is not major histocompatibility complex restricted and that the infused MSC-Exos are immunotolerated by the host, which is convenient for a future clinical application of MSC-Exos in IBD.

Dysregulation of inflammatory mediators and disruption of intestinal barrier integrity in IBD patients and in experimental colitis often interact in a positive feedback loop, together forming a vicious cycle (2931). MSC-Exos treatment could serve dual roles to break this cycle, by reducing a panel of inflammatory mediators and maintaining intestinal homeostasis. Regarding the potential mechanisms, it is interesting that infused MSC-Exos are mainly targeted to macrophages in DSS-elicited inflamed colons. In addition, MSC-Exos treatment increases markers indicative of M2 macrophages in the colons of DSS-colitic mice. Therefore, intestinal macrophages may mediate the anticolitic benefit of MSC-Exos. Indeed, this benefit of MSC-Exos was abolished in macrophage-depleted mice, which further supports our hypothesis.

Subsequently, we sought to understand why pretreatment with MSC-Exos failed to exert prophylactic effects on experimental colitis. By in vivo trafficking MSC-Exos, we found that noninflamed intestines that failed to recruit infused MSC-Exos and macrophages made up only $5 \%-10 \%$ of all the cells that incorporated 
MSC-Exos, indicating an equal distribution in the body. This may provide a straightforward biological explanation for the failure of pretreatment. Furthermore, infused MSC-Exos were detected and maintained in the inflamed colons for no less than 5 days after injection, a time lag long enough to exert long-lasting protective actions. In striking contrast, the presence of transplanted MSCs decreased dramatically in the recipient at 2 days after infusion (32). This may endow MSC-Exos with a therapeutic advantage over MSCs. Noteworthy for MSC-Exos treatment is the possibility that the increase of M2 macrophage activity may lead to intestinal fibrosis, a severe and distressing complication of $\operatorname{IBD}(33,34)$. On this front, although our data negate that MSC-Exos treatment favors intestinal fibrosis, optimization research establishing an ideal model for experimental research of intestinal fibrosis should be performed to monitor the possibility of fibrosis after MSCExos transplantation.

Regulatory cytokines can inhibit murine experimental colitis $(35,36)$. We found that colonic levels of IL-10 were substantially increased in mice treated with DSS plus MSC-Exos compared with DSS plus PBStreated control mice. Moreover, protection from colitis in MSC-Exos-treated mice was dependent on regulatory cytokine IL-10 because administration of MSC-Exos was less effective in DSS-colitic mice treated with $\mathrm{mAb}$ against IL-10. Subsets of T cells are a possible source of the elevated IL-10 (25, 26). However, we observed that MSC-Exos treatment failed to increase the production of IL-10 on ex vivo culture, which preliminarily negates the hypothesis that subsets of $\mathrm{T}$ cells produce the elevated IL-10. In contrast, macrophages isolated from treated DSS-colitic mice produced substantially higher levels of IL-10 than those from untreated mice. These results suggest that colonic macrophages are at least 1 source of the increased IL-10 and reinforce the notion that macrophage-derived IL-10 is a critical mediator of the anticolitic benefit of MSC-Exos. Nevertheless, macrophage-derived IL-7 may be involved in ameliorating murine experimental colitis when treated with exosomes from human umbilical cord MSCs (37). Therefore, we cannot exclude the possibility that additional factors after transfer of MSC-Exos in DSS-colitic mice may also play a role in protection from colitis because neutralization of IL-10 alone did not fully block the anticolitic effect of MSC-Exos.

Infiltration and activation of neutrophils in the intestinal mucosa are essential to the pathogenesis of IBD $(38,39)$. Neutrophils migrate into the lamina propria of DSS-induced colitis, where they eliminate invading bacteria using MPO, concomitantly eliciting oxidative organ damage, an undesirable effect of MPO (40-42). As such, MPO activity has become a widely accepted indicator of neutrophil activity. We observed that colonic MPO activity was significantly decreased in DSS-colitic mice treated with MSCExos, indicating that MSC-Exos treatment inhibits neutrophil invasion. IL-10 is a potent regulator of the migration and invasion of neutrophils. It is possible that the decreased MPO activity was a consequence of the elevated IL-10 in MSC-Exos-treated DSS-colitic mice. This was primitively validated by the fact that the neutralizing anti-IL-10 antibody reversed the reduction of colonic MPO activity following intravenous injection of MSC-Exos.

To assess the molecular basis mediating the effects of MSC-Exos, we performed a proteomic analysis of MSC-Exos, which indicated that immunosuppressive or antiinflammatory activity is a salient feature of MSC-Exos. Specifically, our findings revealed that levels of metallothionein-2 were extremely high in MSC-Exos. When metallothionein-2 was inhibited in the parent MSCs, the antiinflammatory effects of MSC-Exos on macrophages in vitro were significantly suppressed. NF- $\mathrm{BB}$ signaling is a downstream target of metallothionein-2 $(27,43)$. Consistent with this, MSC-Exos induced a significant decrease in NF- $\mathrm{kB}$ activity, whereas this effect was compromised when the expression of metallothionein-2 in MSC-Exos was inhibited. Our findings suggest that metallothionein-2 in MSC-Exos acts as a critical negative regulator of the inflammatory response in macrophages. Of note, metallothionein-2 possesses high conservation between Homo sapiens and Mus musculus, indicating that human metallothionein-2 in MSC-Exos could work in mice. However, the antiinflammatory effects of MSC-Exos were not fully blocked by metallothionein-2 inhibition both in vitro and in vivo, suggesting that other components of MSC-Exos might also serve as active mediators.

Metallothionein-2, a member of the metallothionein family, is a low-molecular weight, cysteine-rich protein $(44,45)$. Metallothioneins are present in multiple tissues and mainly consist of 4 groups: metallothionein-1A, metallothionein-2, metallothionein-3, and metallothionein-4. It has been reported that metallothionein-2 serves as a critical regulator of numerous cell activities, including differentiation, apoptosis, autophagy, immune response, carcinogenesis, and drug resistance (28, 46-50). The effects of metallothionein-2 on pathophysiological processes, particularly inflammation, are subjects of many studies (51). A study by Liu et al. showed that metallothionein-2 knockdown increased LPS-induced 
IL-6 production in endothelial cells (49), indicating a protective role against inflammatory responses. Takano and colleagues demonstrated that transgenic mice with metallothionein overexpression were more resistant to neutrophilic lung inflammation and lung edema induced by intratracheal challenge with LPS (52). Metallothionein-2 has also been found to be a critical regulator of NF- $\mathrm{KB}$ signaling because its upregulation attenuates NF- $\mathrm{B}$ activity in tumor cells, keloid fibroblastic cells, as well as cardiomyocyte cells (53-56). In line with these studies, our results demonstrated the antiinflammatory effects of metallothionein-2 on both macrophages and mouse colitis models. It is noteworthy that the presence of this protein in exosomes may offer a therapeutic advantage over its free form because free proteins are unlikely to interact with cells or have uptake into cells $(57,58)$. Furthermore, the role of metallothionein-2 might not be unique for colitis. In contrast, it should be applicable to other disorders, especially those with macrophages involved in the pathogenesis.

In summary, we propose a novel therapeutic strategy for the treatment of IBD based on the use of MSC-Exos, and of obvious therapeutic significance is that generation of a clinically effective dosage of MSC-Exos under good manufacturing practice is feasible and rapid (59) and that MSC-Exos can be stored frozen and ready to use in any clinical scenario. Once the benefits and risks related to the infusion of MSC-Exos are well evaluated, this cell-free therapy will provide new perspectives for IBD therapy.

\section{Methods}

Detailed procedures are provided in Supplemental Methods.

Exosome preparation. Exosomes were isolated from the supernatant of human bone marrowderived MSCs (herein referred to as MSC-Exos) by standard ultracentrifugation methods, as previously described (60).

Induction of experimental colitis. IBD mouse models of experimental colitis were induced by oral DSS administration and by intrarectal infusion of TNBS. Exosomes were intravenously administrated to mice at the indicated time, and macrophage depletion using Clod-lipos was performed as previously described (61-63). Mice were sacrificed at the indicated time, the entire colon was removed from the cecum to the anus, and colon length was measured as an indirect marker of inflammation. Colon segments were processed for histopathological or flow cytometry analysis or frozen in liquid nitrogen for protein and RNA extraction. Cytokine and MPO activities were measured according to the manufacturer's protocol. To track injected exosomes in vivo, MSC-Exos were labeled with PKH26 before infusion and analyzed via flow cytometry analysis.

Histopathological evaluation. Collected colon samples were fixed with $10 \%$ formalin and embedded in paraffin. After paraffin embedding, 5- $\mu$ m thick sections were made and stained with HE. Histopathology scores were determined in colonic sections by 2 blinded trained pathologists (unaware of group identity), with a combined score for inflammatory cell infiltration (score, 0-3) and tissue injury (score, 0-3), according to our previous report (2).

Isolation of macrophages from the lamina propria of mouse colons. Colonic macrophages were isolated using immunomagnetic separation with anti-F4/80 mAb (Miltenyi Biotec), determined by F4/80 ${ }^{+}$and CD11b staining, and then used for the analysis of cytokine secretion.

Measurement of colonic tissue bacterial load. Colon tissues were collected and homogenized in sterile physiological saline. After centrifuging at $13,000 \mathrm{~g}$ for 10 minutes at $4^{\circ} \mathrm{C}$ to remove insoluble material, $100 \mu \mathrm{L}$ of the tissue lysate supernatants containing $100 \mu \mathrm{g}$ of colonic tissue extracts was plated in duplicate on blood agar plates and incubated at $37^{\circ} \mathrm{C}$ for 2 days. Bacterial loads were defined as the average number of bacterial colony-forming units per gram of colonic tissues (wet weight).

Statistics. Results from the duplicate reproducible experiments were combined for statistical analysis. SPSS16.0 was used for all the statistical analyses. Data represent mean \pm SD. To determine the statistical significance, 2-tailed Student's $t$ test or 1-way ANOVA was applied for the continuous variables with normal distributions, whereas Mann-Whitney or Kruskal-Wallis test was used when distributions were skewed. $P \leq 0.05$ was considered statistically significant.

Study approval. All samples from human tissues were collected with written informed consent from donors, and all procedures were performed with the approval of the Institutional Review Board of The Sixth Affiliated Hospital of Sun Yat-sen University. Animal experiments were approved by the Institutional Animal Care and Use Committee of Sun Yat-sen University and conformed to the Guide for the Care and Use of Laboratory Animals of the National Institutes of Health (National Academies Press, 2011) in China. 


\section{Author contributions}

HL, ZL, FW, XW, and PL conceptualized the study. HL, XH, XW, and PL developed the experimental methods. HL, ZL, FW, CZ, XZ, TH, XW, and PL performed the investigations and data analyses. HL and XW wrote the original draft of the manuscript. HL, ZL, FW, CZ, XZ, TH, XH, XW, AND PL reviewed and edited the manuscript. PL and XW acquired funding. PL and XW supervised the study. HL, ZL, and FW contributed equally to this work. HL, ZL, and FW initiated and completed the work. This determined the authorship order.

\section{Acknowledgments}

This work was supported by National Natural Science Foundation of China (81870383), Science and Technology Planning Project of Guangzhou City (201804010014), Science and Technology Planning Project of Guangdong Province (2015B020229001), Clinical Innovation Research Program of Guangzhou Regenerative Medicine and Health Guangdong Laboratory (2018GZR0201005), Guangdong Natural Science Foundation (2017A030313785), China Postdoctoral Science Foundation (2019TQ0390), National Key R\&D Program of China (2017YFC1308800), and Science and Technology Planning Project of Guangdong Province (20160916).

Address correspondence to: Xianrui Wu and Ping Lan, The Sixth Affiliated Hospital, Sun Yat-sen University, 26 Yuancun Erheng Road, Guangzhou, Guangdong, China, 510655. Phone: 011.86.020.38254009; Email: wuxianr5@mail.sysu.edu.cn (XW); lanping@mail.sysu.edu.cn (PL).

1. Ng SC, et al. Worldwide incidence and prevalence of inflammatory bowel disease in the 21st century: a systematic review of population-based studies. Lancet. 2018;390(10114):2769-2778.

2. Zheng XB, et al. Engulfment and cell motility protein 1 protects against DSS-induced colonic injury in mice via Rac1 activation. J Crohns Colitis. 2019;13(1):100-114.

3. West NR, et al. Oncostatin M drives intestinal inflammation and predicts response to tumor necrosis factor-neutralizing therapy in patients with inflammatory bowel disease. Nat Med. 2017;23(5):579-589.

4. Renna S, Cottone M, Orlando A. Optimization of the treatment with immunosuppressants and biologics in inflammatory bowel disease. World J Gastroenterol. 2014;20(29):9675-9690.

5. Forbes GM, et al. A phase 2 study of allogeneic mesenchymal stromal cells for luminal Crohn's disease refractory to biologic therapy. Clin Gastroenterol Hepatol. 2014;12(1):64-71.

6. Panés J, et al. Expanded allogeneic adipose-derived mesenchymal stem cells (Cx601) for complex perianal fistulas in Crohn's disease: a phase 3 randomised, double-blind controlled trial. Lancet. 2016;388(10051):1281-1290.

7. Duijvestein M, et al. Autologous bone marrow-derived mesenchymal stromal cell treatment for refractory luminal Crohn's disease: results of a phase I study. Gut. 2010;59(12):1662-1669.

8. Van Outryve M, Debongnie JC. GLEM/LOK report on proctology practice in Belgium. Results, comments and recommendations. Acta Gastroenterol Belg. 2006;69(1):25-30.

9. Griffin MD, Elliman SJ, Cahill E, English K, Ceredig R, Ritter T. Concise review: adult mesenchymal stromal cell therapy for inflammatory diseases: how well are we joining the dots? Stem Cells. 2013;31(10):2033-2041.

10. Sala E, et al. Mesenchymal stem cells reduce colitis in mice via release of TSG6, independently of their localization to the intestine. Gastroenterology. 2015;149(1):163-176.e20.

11. Willis GR, et al. Mesenchymal stromal cell exosomes ameliorate experimental bronchopulmonary dysplasia and restore lung function through macrophage immunomodulation. Am J Respir Crit Care Med. 2018;197(1):104-116.

12. Lee C, et al. Exosomes mediate the cytoprotective action of mesenchymal stromal cells on hypoxia-induced pulmonary hypertension. Circulation. 2012;126(22):2601-2611.

13. Monsel A, Zhu YG, Gudapati V, Lim H, Lee JW. Mesenchymal stem cell derived secretome and extracellular vesicles for acute lung injury and other inflammatory lung diseases. Expert Opin Biol Ther. 2016;16(7):859-871.

14. Jeong JO, et al. Malignant tumor formation after transplantation of short-term cultured bone marrow mesenchymal stem cells in experimental myocardial infarction and diabetic neuropathy. Circ Res. 2011;108(11):1340-1347.

15. Phinney DG, Pittenger MF. Concise review: MSC-derived exosomes for cell-free therapy. Stem Cells. 2017;35(4):851-858.

16. Sun Y, et al. Human mesenchymal stem cell derived exosomes alleviate type 2 diabetes mellitus by reversing peripheral insulin resistance and relieving $\beta$-cell destruction. ACS Nano. 2018;12(8):7613-7628.

17. Kordelas L, et al. MSC-derived exosomes: a novel tool to treat therapy-refractory graft-versus-host disease. Leukemia. 2014;28(4):970-973.

18. Liu W, et al. Exosomes derived from bone mesenchymal stem cells repair traumatic spinal cord injury by suppressing the activation of A1 neurotoxic reactive astrocytes. J Neurotrauma. 2019;36(3):469-484.

19. Zhao H, et al. Exosomes from adipose-derived stem cells attenuate adipose inflammation and obesity through polarizing $\mathrm{M} 2$ macrophages and beiging in white adipose tissue. Diabetes. 2018;67(2):235-247.

20. Chen CY, et al. Exosomal DMBT1 from human urine-derived stem cells facilitates diabetic wound repair by promoting angiogenesis. Theranostics. 2018;8(6):1607-1623.

21. Rani S, Ritter T. The exosome - a naturally secreted nanoparticle and its application to wound healing. Adv Mater Weinheim. 2016;28(27):5542-5552.

22. Neurath MF. Cytokines in inflammatory bowel disease. Nat Rev Immunol. 2014;14(5):329-342. 
23. Strober W, Fuss I, Mannon P. The fundamental basis of inflammatory bowel disease. J Clin Invest. 2007;117(3):514-521. 24. Maloy KJ, Powrie F. Intestinal homeostasis and its breakdown in inflammatory bowel disease. Nature. 2011;474(7351):298-306. 25. Moore KW, O'Garra A, de Waal Malefyt R, Vieira P, Mosmann TR. Interleukin-10. Annu Rev Immunol. 1993;11:165-190.

26. Kapur R, et al. T regulatory cells and dendritic cells protect against transfusion-related acute lung injury via IL-10. Blood. 2017;129(18):2557-2569.

27. Lin S, et al. Transcription factor myeloid Zinc-finger 1 suppresses human gastric carcinogenesis by interacting with metallothionein 2A. Clin Cancer Res. 2019;25(3):1050-1062.

28. Li X, et al. Exosome derived from human umbilical cord mesenchymal stem cell mediates MiR-181c attenuating burn-induced excessive inflammation. EBioMedicine. 2016;8:72-82.

29. Tatiya-Aphiradee N, Chatuphonprasert W, Jarukamjorn K. Immune response and inflammatory pathway of ulcerative colitis. $J$ Basic Clin Physiol Pharmacol. 2018;30(1):1-10

30. Wang H, et al. Pro-inflammatory miR-223 mediates the cross-talk between the IL23 pathway and the intestinal barrier in inflammatory bowel disease. Genome Biol. 2016;17:58.

31. Edelblum KL, Yan F, Yamaoka T, Polk DB. Regulation of apoptosis during homeostasis and disease in the intestinal epithelium. Inflamm Bowel Dis. 2006;12(5):413-424.

32. Gonzalez-Rey E, Anderson P, González MA, Rico L, Büscher D, Delgado M. Human adult stem cells derived from adipose tissue protect against experimental colitis and sepsis. Gut. 2009;58(7):929-939.

33. Lawrance IC, et al. Cellular and molecular mediators of intestinal fibrosis. J Crohns Colitis. 2017;11(12):1491-1503.

34. Danese S, et al. Identification of endpoints for development of antifibrosis drugs for treatment of Crohn's Disease. Gastroenterology. 2018;155(1):76-87.

35. Hayashi A, et al. A single strain of Clostridium butyricum induces intestinal IL-10-producing macrophages to suppress acute experimental colitis in mice. Cell Host Microbe. 2013;13(6):711-722.

36. Qiu X, Zhang M, Yang X, Hong N, Yu C. Faecalibacterium prausnitzii upregulates regulatory T cells and antiinflammatory cytokines in treating TNBS-induced colitis. J Crohns Colitis. 2013;7(11):e558-e568.

37. Mao F, et al. Exosomes derived from human umbilical cord mesenchymal stem cells relieve inflammatory bowel disease in mice. Biomed Res Int. 2017;2017:5356760.

38. Segal AW. The role of neutrophils in the pathogenesis of Crohn's disease. Eur J Clin Invest. 2018;48 Supp1 2:e12983.

39. Zhou G, et al. CD177 ${ }^{+}$neutrophils as functionally activated neutrophils negatively regulate IBD. Gut. 2018;67(6):1052-1063

40. Farooq SM, Stillie R, Svensson M, Svanborg C, Strieter RM, Stadnyk AW. Therapeutic effect of blocking CXCR2 on neutrophil recruitment and dextran sodium sulfate-induced colitis. J Pharmacol Exp Ther. 2009;329(1):123-129.

41. Kolaczkowska E, Kubes P. Neutrophil recruitment and function in health and inflammation. Nat Rev Immunol. 2013;13(3):159-175.

42. Matthijsen RA, et al. Myeloperoxidase is critically involved in the induction of organ damage after renal ischemia reperfusion. Am J Pathol. 2007;171(6):1743-1752.

43. Pan Y, et al. Epigenetic upregulation of metallothionein 2a by diallyl trisulfide enhances chemosensitivity of human gastric cancer cells to docetaxel through attenuating NF-kB activation. Antioxid Redox Signal. 2016;24(15):839-854.

44. Patankar HV, et al. Overexpression of a Metallothionein $2 A$ gene from date palm confers abiotic stress tolerance to yeast and Arabidopsis thaliana. Int J Mol Sci. 2019;20(12):E2871.

45. Raudenska M, et al. Levels of heavy metals and their binding protein metallothionein in type 2 diabetics with kidney disease. J Biochem Mol Toxicol. 2017;31(6).

46. $\mathrm{Ma} \mathrm{H}$, et al. HMBOX1 interacts with MT2A to regulate autophagy and apoptosis in vascular endothelial cells. Sci Rep. 2015;5:15121.

47. Habel N, et al. Zinc chelation: a metallothionein 2A's mechanism of action involved in osteosarcoma cell death and chemotherapy resistance. Cell Death Dis. 2013;4:e874

48. Azuma Y, Chou SC, Lininger RA, Murphy BJ, Varia MA, Raleigh JA. Hypoxia and differentiation in squamous cell carcinomas of the uterine cervix: pimonidazole and involucrin. Clin Cancer Res. 2003;9(13):4944-4952.

49. Liu Y, Liu H, Chen W, Yang T, Zhang W. EOLA1 protects lipopolysaccharide induced IL-6 production and apoptosis by regulation of MT2A in human umbilical vein endothelial cells. Mol Cell Biochem. 2014;395(1-2):45-51.

50. Canpolat E, Lynes MA. In vivo manipulation of endogenous metallothionein with a monoclonal antibody enhances a T-dependent humoral immune response. Toxicol Sci. 2001;62(1):61-70.

51. Inoue K, Takano H, Shimada A, Satoh M. Metallothionein as an antiinflammatory mediator. Mediators Inflamm. 2009;2009:101659.

52. Takano H, et al. Protective role of metallothionein in acute lung injury induced by bacterial endotoxin. Thorax. 2004;59(12):1057-1062.

53. Pan Y, et al. Metallothionein 2A inhibits NF-кB pathway activation and predicts clinical outcome segregated with TNM stage in gastric cancer patients following radical resection. J Transl Med. 2013;11:173

54. Marikar FM, Jin G, Sheng W, Ma D, Hua Z. Metallothionein 2A an interactive protein linking phosphorylated FADD to NF-кB pathway leads to colorectal cancer formation. Chin Clin Oncol. 2016;5(6):76

55. Toh PP, et al. Modulation of metallothionein isoforms is associated with collagen deposition in proliferating keloid fibroblasts in vitro. Exp Dermatol. 2010;19(11):987-993.

56. Cong W, et al. Metallothionein prevents age-associated cardiomyopathy via inhibiting NF-kB pathway activation and associated nitrative damage to 2-OGD. Antioxid Redox Signal. 2016;25(17):936-952.

57. Gonda A, Kabagwira J, Senthil GN, Wall NR. Internalization of exosomes through receptor-mediated endocytosis. Mol Cancer Res. 2019;17(2):337-347.

58. Kornilova ES. Receptor-mediated endocytosis and cytoskeleton. Biochemistry Mosc. 2014;79(9):865-878.

59. Mendt M, et al. Generation and testing of clinical-grade exosomes for pancreatic cancer. JCI Insight. 2018;3(8):99263.

60. Théry C, Amigorena S, Raposo G, Clayton A. Isolation and characterization of exosomes from cell culture supernatants and biological fluids. Curr Protoc Cell Biol. 2006; Chapter 3:Unit 3.22.

61. van Rooijen N, Bakker J, Sanders A. Transient suppression of macrophage functions by liposome-encapsulated drugs. Trends 
Biotechnol. 1997;15(5):178-185.

62. Anthony RM, et al. Memory $\mathrm{T}(\mathrm{H}) 2$ cells induce alternatively activated macrophages to mediate protection against nematode parasites. Nat Med. 2006;12(8):955-960.

63. Hunter MM, et al. In vitro-derived alternatively activated macrophages reduce colonic inflammation in mice. Gastroenterology. 2010;138(4):1395-1405. 